\title{
L'histoire du kava commence par sa découverte
}

Vincent Lebot

\section{Citer ce document / Cite this document :}

Lebot Vincent. L'histoire du kava commence par sa découverte. In: Journal de la Société des océanistes, 88-89, 1989-12. pp. 89-114.

doi : $10.3406 /$ jso.1989.2855

http://www.persee.fr/doc/jso_0300-953x_1989_num_88_1_2855

Document généré le 16/10/2015 


\section{L'histoire du kava commence par sa découverte}

par

Vincent LEBOT*

\section{INTRODUCTION}

Dès leurs premières enquêtes, les explorateurs ont voulu savoir de quelles façons les indigènes avaient accès aux "paradis artificiels ". Leurs observations indiquent qu'aucune des espèces végétales habituellement destinées à cet usage n'étaient présentes en Océanie (Cannabis indica, Erythroxylon coca, Datura spp., Papaver somniferum...), de même que les insulaires ne connaissaient pas l'usage de l'alcool (Forster, 1786). Par contre, ces observations indiquent et mentionnent l'existence d'une III houvelle plante inconnue jusque là : Le kava (Piper methysticum Forst. f.). Le terme kava provient du polynésien ava qui signifie boisson nébriante (Steinmetz, 1960). Il ne s'agit ni d'un nallucinogène, puisque la perception des conditions normales de la réalité n'est pas altérée, ni d'un stupéfiant puisque le kava ne provoque pas d'inhibition du système nerveux central ni d'accoutumance ou de dépendance. La précision est importante, car elle permet de mieux comprendre l'esprit convivial qui entoure l'absorption du breuvage et donc le rôle socioculturel que joue le kava au sein des communautés insulaires, comparable en tous points à celui du vin. Ce rôle a évolué au cours des âges. Présent dans la quasi-totalité des îles du Pacifique au début de l'ère européenne, le kava a dû affronter les interdits religieux et parfois le pouvoir colonial, aujourd'hui responsables de sa disparition dans de nombreuses îles. Traditionnellement utilisé comme offrande coutumière, boisson rituelle et plante médicinale, il tend à devenir une boisson conviviale de consommation quotidienne. Celle-ci connaît un réel développement en raison de la prise de conscience de la part des sociétés océaniennes de leur identité culturelle et de leur volonté de l'affirmer, mais aussi du fait de ses qualités et propriétés propres qui le distinguent des autres boissons auxquelles ont pourtant accès les insulaires.

Le kava est aussi une caractéristique de l'Océanie car il s'agit de la seule espèce cultivée de cette importance économique dont l'aire de distribution est exclusivement limité à cette zone géographique. La plupart des botanistes qui se sont interessés au $P$. methysticum se sont posé la question de son origine et de sa surprenante distribution (Brown, 1935 ; Yuncker, 1959 ; Barrau, 1962 ; Smith, 1981). Quelle est l'origine géographique de cette espèce? Les avis sont partagés mais rares sont les conclusions définitives. Trouver l'origine d'une plante cultivée est pourtant un exercice aussi difficile qu'utile. Parce que le kava est essentiel pour ces sociétés, suivre les déplacements du kava c'est aussi suivre les hommes.

Il semble que les données disponibles aujourd'hui permettent de résoudre cette énigme d'ethnobotanique océanienne. Le présent article vise à faire le point sur les résultats obtenus. L'approche adoptée pour étudier le Kava est pluridisciplinaire et résulte de la nécessité d'observer les différents aspects d'une culture traditionnelle, de retourner le problème de tous les côtés pour mieux le comprendre et pour finalement mieux appréhender cette plante qui, plus qu'une simple espèce végétale est un véritable phénomène de société. L'étude pluridisciplinaire est très précieuse. Elle permet dans bien des occasions de s'assurer que les résultats obtenus à l'aide d'une méthode particulière ne sont pas contredits par une autre. Il s'agit en fait, de vérifier que les hypothèses formulées restent tangibles quand on aborde le problème d'une autre façon. L'origine du kava sera donc discutée et argumentée en fonction des données connues sur lintroduction des plantes cultivées en Océanie. Mais aussi d'après la tradition

* Horticulture Department. University of Hawaii. Honolulu. 
orale, la mythologie et les classifications vernaculaires des différents "crus" et " cépages". Les informations obtenues sont confirmées à l'aide de méthodes botaniques, génétiques et chimiques.

\section{L'ORIGINE ET LA DISTRIBUTION DES PLANTES CUltivéEs EN OCÉANIE}

Lorsque les Australoïdes arrivent sur la terre de Sahul il y a 40000 ans, ils y découvrent une flore très différente de celle du sous-continent de la Sonde. Ils viennent en fait de traverser la ligné Huxleý (dérivée de la célèbre ligne'Wallacé) qui constitue la frontière bio-géographique entre le sous-continent de la Sonde et l'Océanie. De part et d'autre de cette limite la faune et la flore diffèrent considérablement en raison de l'isolement qui caractérise Sahul à cette époque (Bellwood, 1981). Ces chasseurscueilleurs australoïdes vont se sédentariser puis domestiquer des espèces sauvages et les cultiver, ils inventent ainsi l'horticulture océanienne. Il est d'ailleurs probable qu'ils soient à l'origine des premières formes connues de l'agriculture et ce, bien avant l'apparition du foyer cultural mésopotamien (Barrau, 1956; Bellwood, 1981 ; Bonnemaison, 1986; Yen, 1988).

Pour Vavilov (1951), les centres d'origine des plantes cultivées sont localisés géographiquement. Le centre d'origine indo-malais comprend, selon lui, la péninsule malaise, les Philippines et l'Indonésie. Harlan (1970) pense plutôt que le non-centre d'origine indo-malais, qu'il nomme B2, s'étend de la péninsule indienne à la Papouasie-Nouvelle-Guinée. Curieusement, ce dernier auteur fait fi de la frontière bio-géographique de la ligne 'Wallacé dont Bellwood (1981) souligne pourtant l'importance et qui sépare les types biologiques en deux entités distinctes, indo-malaise et australo-océanienne. Haudricourt et Hedin (1943) et Barrau (1965) pensent eux aussi qu'il conviendrait de parler d'un centre indo-océanien qui s'étendrait jusqu'à la Mélanésie occidentale. Pour les généticiens en général, les îles d'Océanie ne font pas partie d'un des centres d'origine et de domestication des plantes cultivées. Les îles océaniques émergent brûlées par le magma qui les stérilise. La vie des îles a donc commencé ailleurs, sur les continents, et il faut plusieurs siècles pour qu'une végétation s'y installe au bénéfice des spores et graines véhiculées par les vents ou les oiseaux. Cette végétation est souvent très pauvre car les espèces capables de tels voyages au dessus de l'océan sont peu nombreuses. Bien que les îles océaniques présentent des taux d'endémisme très élevés pour les genres et espèces qui y poussent à l'état spontané, il n'existe pas de plante cultivée qui soit originaire de ces îles si l'on en juge par l'état des données disponibles. D'une manière générale, plus une île est éloignée d'une masse continentale, plus le nombre d'espèces est faible et plus le taux d'endémisme est élevé.

Plusieurs auteurs se sont intéressés à la qucstion et ont montré, à l'aide de méthodes diverses, que la plupart des cultures traditionnelles d'Océanie avaient la Papouasie-Nouvelle-Guinée ou l'arc mélanésien pour centre d'origine et de domestication. C'est le cas notamment de certains taros (Hornell, 1946), de l'arbre à pain (Wilder, 1928 et Barrau, 1962), de la banane $f e^{\prime} h i$ (Mac Daniels, 1971), du cocotier, de la canne à sucre et de certains ignames (Barrau, 1965). De toutes les espèces cultivées par les Océaniens avant l'arrivée des Européens, seule la patate douce n'est pas issue de ce centre de domestication. Barrau (1957) et Yen (1974) ont discuté les diverses hypothèses avancées pour expliquer la présence de cette espèce d'origine américaine en Océanie, nous n'y reviendrons pas.

Il y a près de 4000 ans, quand les Mélanésiens arrivent dans l'île d'Anatom située au sud de l'archipel de Vanuatu, le cocotier (Cocos nucifera) y est déjà installé depuis 2000 ans et constitue une ressource alimentaire inespérée pour ces migrants (Spriggs, 1982). Le phénomène s'est probablement répété ailleurs, notamment en Polynésie, car cette espèce est la seule plante alimentaire de cette importance qui puisse voyager plusieurs mois au gré des courants marins pour coloniser les îles océaniques.

À part le cocotier, la quasi-totalité des espèces traditionnellement cultivées à l'ère préeuropéenne en Polynésie étaient reproduites à l'aide de boutures ou de fractions de tubercules. Tel est le cas des taros (Amorphophallus campanalatus, Alocasia macrorrhyza, Colocasia esculenta et $C$. antiquorum, Cyrtosperma chamissonis), des ignames (Dioscorea esculenta, $D$. bulbifera, $D$. pentaphylla, $D$. nummularia, $D$. alata), de la patate douce (Ipomea batatas), de l'arbre à pain (Artocarpus altilis), des bananiers (Musa paradisiaca M. troglodytarum, M. sapientum), de la canne à sucre (Saccharum officinarum) et du kava (Piper methysticum). Lorsque les premiers habitants arrivent sur ces îles, ils ont avec eux leurs plantes traditionnelles car il y a de leur survie. La dispersion des espèces cultivées en Océanie a donc suivi les courants de migrations. Les grands catamarans utilisés par les navigateurs polynésiens comportaient 
un jardin (Garanger, 1972 ; Bellwood, 1981 ; Bonnemaison, 1986). Les cultures y étaient conservées vivantes tout au long de la traversée puisqu'en l'absence de graines, seule la multiplication végétative permettait la reproduction de ce matériel végétal à l'arrivée.

\section{L'AIRE DE DISTRIBUTION DU KAVA}

En Micronésie, le kava est bu à Ponapé où on le nomme Sakau (il était aussi consommé sur l'île voisine de Kosrae au début du siècle Riesenberg, 1968).

En Polynésie, il semblerait que toutes les îles hautes, à l'exception de la Nouvelle-Zélande, de l'île de Pâques et de Rapa, en aient connu la consommation et la culture à un moment ou un autre de leur histoire (Marshall, 1976; Gatty, 1956). À Wallis et Futuna, il est connu sous le nom de kava, aux îles Samoa : $A v a$, à Tonga : kava. Aux îles Hawaï, où on ne le cultive plus : $A w a$, aux îles Marquises : Kawakawa, à Niue : Kavaïnu, à Tubuai : $A v a$, et à Tahiti : Ava, Ava-Ava ou Evava (Cuzent, 1857). Dans toute la partie de l'Océanie où la présence du Kava semble liée à une introduction d'origine polynésienne, (îles de Polynésie mais aussi sud de l'archipel de Vanuatu : kava sur l'île de Tanna) la plante est connue sous divers vocables d'affinité polynésienne.

En Mélanésie, il est connu sous le nom de Yagona à Fidji et n'a jamais été cultivé en Nouvelle-Calédonie. À Vanuatu, le kava est connu sous le même nom en Bichlamar (langue véhiculaire à base lexicale anglaise). Mais il est dénommé différemment dans la plupart des nombreuses langues vernaculaires de Vanuatu. Bien qu'il soit aujourd'hui cultivé dans toutes les îles de l'archipel, il ne semble pas que cela ait toujours été le cas. Les récits et observations diffèrent considérablement d'une île à l'autre (Rivers, 1914; Lester, 1941). Aux îles Salomon, toujours d'après Rivers, le kava était bu à Vanikoro et à Utupua, alors que selon Codrington (1891) et Thompson (1908) il serait inconnu aux îles Santa Cruz. Fox (1924) affirme l'avoir observé à San Cristobal. Un peu plus loin à Anuta, Firth (1954) observe que le kava n'est pas cultivé et n'a jamais été identifié sur l'île.

En Papouasie-Nouvelle-Guinée, la consommation de kava a toujours été très localisée et la situation n'a guère changé aujourd'hui. Le kava est cultivée sur les îles de Baluan et de Karkar, sur la côte de Madang et dans le delta de la rivière Fly. Plus de 800 langues vernaculaires sont toujours vivantes en PapouasieNouvelle-Guinée et le kava est dénommé dans certaines d'entre elles. Dans la plupart de ces langues, le terme utilisé pour désigner le kava signifie tout simplement " racine ", ce qui suggèrerait qu'il fut introduit (comm. pers. Départment of Linguistics, University of Lae, 1987). En Pidgin, langue véhiculaire, le kava est connu sous le nom de Koniak.

Sterly (1970) a montré que dans certaines régions de Papouasie-Nouvelle-Guinée, le kava et le bétel sont consommés ensemble. Tel est le cas aujourd'hui dans les îles de Baluan (Manus) et Karkar (Madang), mais aussi sur la côte Maclay près de Madang et dans la province occidentale, à l'embouchure de la rivière Fly. Les aires de consommation et de culture du kava en Papouasie, se situent toujours sur la côte, jamais à l'intérieur des terres ou dans les zones montagnardes.

On le nomme Kau sur l'île de Baluan dans l'archipel de l'Amirauté (Manus). Observation intéressante, cette île est la seule avec Ponapé où les racines de kava sont écrasées sur une large pierre de basalte et on nomme le kava Sakau à Polapé, ce qui autorise à penser que la plante ou son mode de préparation aurait pu être introduite en Micronésie depuis les îles de l'Amirauté, plutôt que depuis les îles de Polynésie.

Sur la côte Maclay, on le nomme $\mathrm{Keu}$, sur l'île de Karkar en face de Madang, $A y$ you, chez les Samo du village de Nomad dans la province occidentale, $O y o$. Cette localisation du kava en Papouasie-Nouvelle-Guinée est très restrictive géographiquement : on le trouve dans un village mais il est inconnu dans un autre distant de seulement quelques kilomètres. E. E. Henty, ex-conservateur de l'Herbier de Lae. qui a prospecté pendant plus de vingt ans dans ce pays suggère que la plante aurait été introduite dans la province occidentale ("When missionary work began, early this century, catechists, recruited and trained in Tonga and Fidji, were employed. Possibly one of those people took the root to Daru or one of the other station". Henty, 1988, comm. pers.). Barrau (1957) avait déjà observé que des «... auxiliaires Samoans de la Société des missions de Londres ont ainsi introduit jusqu'en Papouasie des clones d' $A$. altilis originaires des Samoa ". Cependant, Maclay a très précisément décrit la consommation de Keu à Astrolabe Bay en 1872, la plante était donc déjà connue dans cette région avant l'arrivée des Européens.

$S$ 'il est relativement aisé de dresser la carte des zones. actuelles et passées de culture de la plante, il est plus difficile de l'expliquer. La multiplication des cultivars de Piper methysticum se fait exclusivement par voie végétative à laaide de boutures de tige. la dispersion géogra- 
phique du kava résulte donc toujours de l'intervention de l'homme qui en véhicule en même temps le nom. La dispersion du kava nous semble plus confuse et plus atomisée en Mélanésie qu'en Polynésie. En effet, la consommation de cette plante, et donc sa culture, y sont curieusement distribuées. Le phénomène peut s'expliquer en partie par la grande disponibilité de superficies à cultiver, mais aussi sans doute par un bien plus grand cloisonnement culturel en Mélanésie. Il pourrait aussi être la conséquence d'un abandon de l'usage du kava dans certaines régions, suivi de sa reprise à la suite de nouveaux contacts. La distribution de la consommation de kava est une énigme qui n'a cessé de passionner les ethnologues dès les premières découvertes (Williams et Calvert, 1859 ; Mikloucho-Maclay, 1886 ; Hough, 1905) mais c'est très certainement Rivers qui en 1914, lorsqu'il publie "The History of Melanesian Society", avance les hypothèses les plus intéressantes quoique très controversées. Rivers pense que le kava fit partie des premières plantes cultivées introduites par les Mélanésiens. Les îles de Mélanésie auraient été peuplées en deux vagues successives de migrants, les "kava-people" d'abord, les "betel-people " ensuite. Les premiers seraient les ancêtres des Vanuatais, les seconds, des Salomonais.

Un inventaire des échantillons existants dans les plus grands herbiers du monde, permet de préciser cette aire de distribution. Plus de 240 échantillons de Piper methysticum ont été récoltés dans le Pacifique, ils proviennent des îles suivantes :

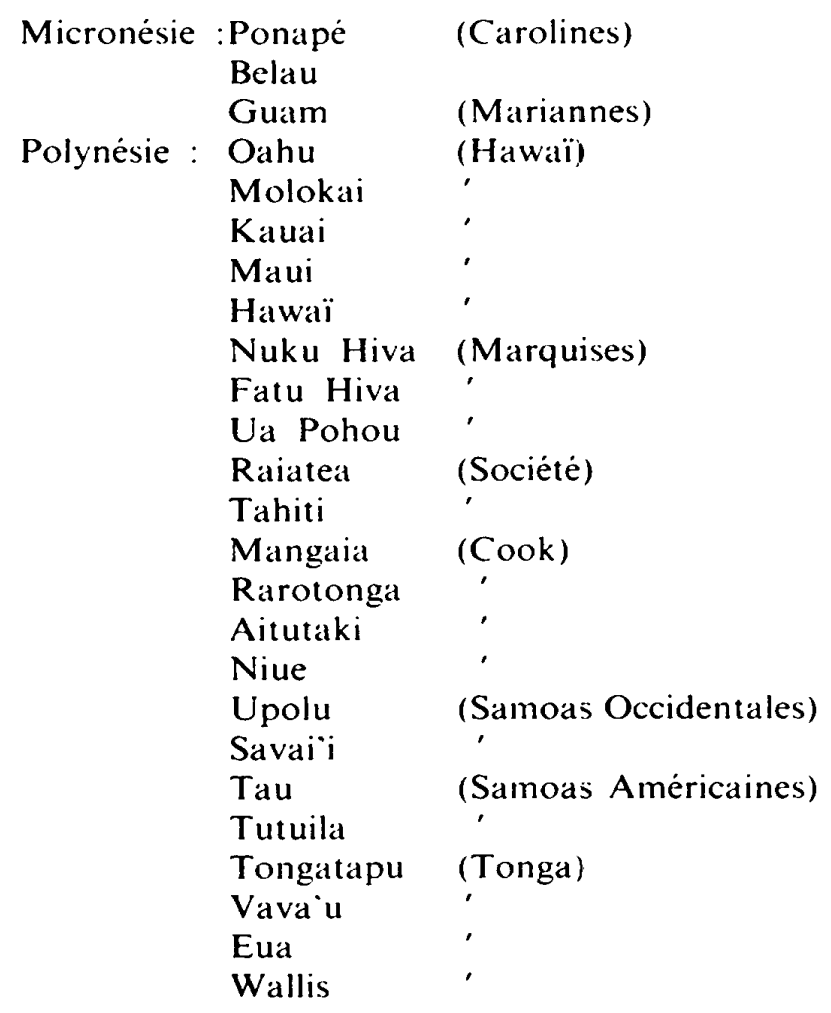

$\begin{array}{ll}\text { Futuna } & , \\ \text { Alofi } & , \\ \text { Mélanésie : Vanua Levu (Fidji) } \\ \text { Viti Levu } \\ \text { Vanua Balavu' } \\ \text { Lakeba } \\ \text { Rewa } \\ \text { Tanna } \\ \text { Anatom } \\ \text { Pentecôte }\end{array}$

En Papouasie-Nouvelle-Guinée 13 échantillons seulement ont été récoltés. Ils proviennent de la province occidentale, du lac Kutubu et de la région de Madang. 3 échantillons sont originaires de l'Irian Jaya mais ont été récoltés à la frontière (sud) avec la Papouasie. Aucun échantillon de $P$. methysticum n'a jamais été récolté aux îles Salomon ou en Nouvelle-Calédonie. Il est surprenant d'apprendre de la part de Forster (1786) qu'il y fut observé lors de son passage dans ce territoire. Quant à Bourgarel (1865), ses affirmations ne se rapprochent en rien de la réalité :

Le kava existe dans quelques points de lîle, mais je ne sache pas que les naturels connaissent ses propriétés et qu'ils en aient jamais prćparé une liqueur enivrante" (p. 403).

Jusqu'à preuve du contraire, l'aire de distribution du kava est exclusivement limitée à ces îles et le $P$. methysticum n'existe nulle part ailleurs sur la planète.

Il est probable que le kava fut introduit partout où l'homme a posé pied à terre. S'il a disparu de nombreuses îles, c'est sous les pressions religieuses exercées par les Missions au début de leur histoire dans le Pacifique. On peut supposer que si le kava n'existait pas dans une île d'Océanie à cette époque, c'est qu'il n'y avait jamais été introduit ou que l'environnement empêchait sa culture. Si le climat de Nouvelle-Zélande avait été favorable à sa croissance, on le retrouverait certainement dans ce pays aujourd'hui. D'ailleurs les ancêtres des Maoris ont appellé Kaw'a-kawa le Macropiper excelsum Forst. (dont ils ne peuvent malheureusement tirer le breuvage. Steinmetz, 1960), en souvenir très certainement des kava qu'ils tentèrent d'y cultiver. Dans la mesure où la présence du kava semble toujours résulter de l'intervention de l'homme, l'approche linguistique permet de délimiter deux zones principales. Celle où la plante s'appelle "kava ", en Polynésie et jusqu'au sud de l'archipel de Vanuatu et où sa présence résulte des migrations des navigateurs polynésiens. Celle où le kava porte des noms génériques mélanésiens, à Fidji, au nord de Vanuatu et en Papouasie-Nouvelle-Guinée. 


\section{LES MYTHES ET LÉGENDES SUR L'ORIGINE DU} KAVA

D'après la tradition orale, l'histoire du kava commence par sa découverte. Les légendes sur l'origine du kava sont nombreuses et toutes le font naître d'un corps humain enterré. Ceci n'est pas propre au kava. L'arbre à pain, le cocotier, l'igname et le taro trouvent tous leurs origines de la même façon (Koenig et al., 1986). En Océanie, les mythes tendent à confirmer que l'homme arrive toujours avant les plantes cultivées sur les îles. Sur les continents, il ne semble pas en être de même, la plante existe avant l'homme et la structure du mythe est différente.

\section{Vanuatu :}

Selon la tradition orale, il existerait deux origines distinctes du kava. Dans les îles du nord de l'archipel, les différentes communautés s'accordent pour situer son origine dans l'île de Maewo.

"Il y a très longtemps, des jumeaux orphelins, un frère et sa sœur, vivaient heureux dans l'île de Maewo. Le garçon qui aimait sa sœur passionnément dut un jour la défendre contre un étranger qui la demandait en mariage et à qui elle fut refusée. Durant la bagarre, l'étranger lui décocha une flèche qui atteignit la sœur et la tua. Le garçon. triste et désespéré, ramena le corps de sa sœur à la maison. lui creusa une tombe et l'enterra. Une semaine après. avant même que les mauvaises herbes ne recouvrent la tombe, apparut une plante qu'il ne connaissait pas et qui lui parut très étrange. Celle-ci poussait. seule sur la tombe. Il décidé de ne pas l'arracher. Un an s’était déjà écoulé sans que le garçon ait pu apaiser la douleur provoquée par le décès de sa sour. Il allait régulièrement se recueillir sur sa tombe. Un jour, il vit un rat tomber raide mort après avoir rongé une des racines de la plante. Il eut alors l"idée de se suicider en absorbant de grandes quantités de ces racines. Mais au lieu de mourrir. il oublia tous ses soucis, renouvela l'opération régulièrement et enseigna l'utilisation de cette plante aux autres hommes. C'est ainsi quapparut pour la première fois le kava"

(version recueillie par V. Lebot à Naviso en Février 1982. côte est de l'île de Maewo, citée dans Lebot et Cabalion, 1986).

Les habitants de l'île de Pentecôte nuancent quelque peu ce mythe en précisant que les premiers kavas consommés provoquaient la nausée mais que la culture et les années aidant, le kava des ancêtres serait devenu beaucoup plus agréable. On retrouve une idée similaire dans les mythes du sud de l'archipel.

Selon les traditions orales des îles du sud, comme Tanna ou Futuna, le kava n'a pas surgi spontanément du corps d'une femme enterrée, mais serait arrivé sur l'île en provenance de l'est. Le chef coutumier du nom de Siaka, du village de Henamanu, au sud-est de l'île de Tanna, raconte le mythe suivant :

"Il y a fort longtemps, les habitants de l'île ne consommaient que du kava sauvage. lorsqu un jour. une femme originaire de Futuna se trouva seule en train d’éplucher ses ignames. Elle était accroupie dans leau, quand le diable profita de l'occasion pour lui glisser un caillou magique dans le vagin. Dès qu'elle s'en aperçut. elle le retira et l'examina. Très intriguée par la forme allongée de celui-ci et par la présence de nouds et de bourgeons. elle décida aussitôt de le rapporter au village. Le chef coutumier en prit possession et l'amena le soir même au nakamal où se trouvaient tous les hommes du village. Alors que ceux-ci entouraient le chef pour observer le caillou, un diable apparut. Il leur montra un kava gros comme un banian et leur expliqua qu il s’agissait là du vrai kava. Il dit aussi que ce caillou était sacré et qu'ils devraient désormais le respecter. Aussitôt dit, ils installèrent le caillou dans une pirogue sculptée dans un bois magique et larrosèrent d'eau. Le lendemain matin. la pirogue était pleine a ras bord de milliers de cailloux identiques. Les gens accoururent de tous les coins de l'ile pour rapporter dans leurs villages ces pierres qui leur permettent aujourd'hui de faire pousser le ka'a grâce à leurs pouvoirs sacrés et magiques".

(version recueillie par V. Lebot auprès du chef Siaka du village de Henamanum, au sud-est de lîle de Tanna en Mai 1982, citée dans Lebot et Cabalion. 1986).

Selon l'explication du monde que donne la tradition orale de l'île de Tanna, les premiers habitants arrivèrent avec l'igname, le taro, l'arbre à pain, le bananier, les choux canaques et les volailles. Ce n'est que bien plus tard que serait arrivée par l'est et par l'île de Futuna, une nouvelle pirogue envoyée par le dieu Karapanemum. Elle apportait sous la forme de pierres magiques trois nouveaux pouvoirs sur Tanna : les cochons, les nouvelles magies noires d'empoisonnement et le kava (Bonnemaison, 1985).

Les îles du sud et du centre de l'archipel subirent des influences culturelles des Tongiens et des Samoans à partir de la Polynésie occidentale, entre les $\mathrm{X}^{\text {" }}$ et $\mathrm{XII}$ ' siècles après $\mathrm{J}$. C. (Garanger, 1972). Le célèbre Roymata à qui l'on doit l'institution d'une chefferie à titres sur les îles du centre de l'archipel, fut l'un de ces conquérants venus de l'est dans ce que les archéologues ont appelé les "phénomènes de retour" (Bonnemaison, 1986).

Pour les gens de Tanna, lorigine locale du kava serait liée à un apport polynésien venu des Samoa ou de Tonga. Les traditions orales sont même bien plus explicites puisqu'elles afiirment que le kava, en même temps que le cochon, fut 
introduit à partir de l'île de Tongatapu. Tanna était en relation maritime avec Tonga et les Tongiens organisaient des voyages en pirogues jusqu`à Futuna où ils venaient se pourvoir en femmes (Bonnemaison, comm. pers., 1987). Aujourd'hui encore le nom utilisé en langues vernaculaires des îles de Tanna et de Futuna pour désigner cette plante est kava, le même qu’à Tonga.

\section{Fidji :}

La tradition orale de l'île de Vanua Levu rapporte que le premier plant de kava est apparu sur la tombe du prince Rangonna peu de temps après son enterrement. Un rat qui s'était précipité pour en consommer les racines devint ivre et servit d'exemple aux hommes. C'est en mémoire du défunt que l'on baptisa cette nouvelle plante Yagona (prononcer yangonna), (Hocart, 1952 et Sterly, 1970).

\section{Samoa :}

Sur l'île de Upolu, les habitants de la baie de Fagalooa assurent que c'est Tagaloa le premier Matai ou chef, qui est à l'origine de son introduction :

"Tagaloa avait deux fils Ava et Sa"a. Ava était mourant lorsquil murmura a Sa a qu une plante de très grande importance pour les Samoans pousserait sur sa tombe. Ava mourut et fut enterré aussitôt. Sa a resta immobile. les yeux fixés sur la tombe. Le troisième jour. deux plantes sortirent de terre a lendroit où se trouvait la tête de Ava. Alors que Sa a regardait, un rat se précipia pour ronger les racines de la premiere plante puis labandonna pour la seconde. Quelques instants apress. Sala observa que le rat titubait d'ivresse. La première plante fut baptisé Tolo. ou canne a sucre. et la deuxieme Ara en mémoirc du défunt".

(version recueillie par $V$. Lebot a Fagalooa. au nordest de l'île d'Upolu aux Samoas occidentales. en mai 1987).

\section{Tonga :}

Gatty (1956) rapporte une légende dont la trame est similaire aux précédentes :

"... on the island of Euaiki, the chief Locul recognized human flesh at a meal and told the people not to eat it it should be planted in the ground and brought to him when it matured into a plant... the body grew up into a kava plant arising from different parts of the body. And when it matured he noticed that a rat chewed on the kara and became paralysed..."

\section{Hawaï :}

La tradition orale des îles Hawaï précise que le kava y fut introduit en provenance de Tahiti et planté pour la première fois sur l'île d'Oahu (Fornander, 1919 : Titcomb, 1948 : Gatty, 1956) :

"Ewa had the courage to test its effect... Ewat said: let me first eat of this plant, and should I die. do not plant it, for it would be valueless: but should I not die, then we will be rich... When Ewa ate it, she became drunk and was intoxicated all day. When she awoke she called this plant $A$ wa the Awa from Kaumakahea, the chief ".

\section{Ponapé :}

Le kara y aurait été introduit secrètement par une femme el en provenance de Kosrae. Pour Ashby (1987):

"... in one legend. the discovery of Saka" is attributed to a rat seen nibbling a root and acting quite intoxicated. In another legend. more detailed. the original is traced to Pohnpeian god $L u k$. The skin of the heel of a mortal man. Uitannar was given by Kuk to a woman in payment for her kindness. She was told to burry the skin and a plant would grow in its place. The juice of the plant would make people intoxicated and change their lives. This was done and the Sakau plant was later spread throughout Pohnpei ".

\section{Papouasie-Nouvelle-Guinée :}

Dans le sud-ouest de la province occidentale, chez les Marind-Anim, le kava serait issu des poils d'un démon --. cigogne dont les pattes ressembleraient aux tiges noueuses de la plante (Nevermann, 1938 d'après Sterly, 1967). Près du lac Kutubu, le kava serait sorti de terre là où séjournait "l'homme souterrain" Waki. Selon d'autres versions c'est le "grand homme " Sagainya lui même qui serait sorti de terre sous forme de kava (Williams, 1940 d'après Sterly, 1967).

Il est intéressant de constater que bien que ces mythes proviennent de sociétés ethniquement différentes, très isolées et situées à des milliers de kilomètres les unes des autres, ils partagent tous une idée commune, ce qui tendrait à montrer une origine commune. Bien que la trame soit souvent la même : d'un corps humain enterré. jaillit un plant de kara dont les racines auraient été consommées pour la première fois par un rat, on observe que ces mythes se répartissent en deux groupes : ceux qui font naître la plante d'un corps humain enterré et ceux qui attribuent son introduction à un être vivant, réel ou mythique. On constate aussi que tous les mythes du premier groupe 
font mention du rat, tandis que ceux du second l'ignorent.

On peut tenter d'examiner la part de vrai qui s'associe à de telles légendes. Les rats sont effectivement attirés par l'odeur que dégagent les racines de kava et il n'est pas rare de trouver des plants attaqués par ces rongeurs. La remarque vaut également pour les cochons sauvages qui dans certaines îles occasionnent des dégâts considérables aux plantations.

Les rats jouent souvent un rôle important dans la mythologie d'Océanie (Wodszicki, 1979). Le rat polynésien, Rattus exulans Peale, originaire d'Asie du sud-est, fut introduit dès les premières migrations et n'occasionna pas de bouleversement notable, il vivait en équilibre semble-t-il avec l'homme et le milieu. Ce n'est que bien plus tard, lors des contacts européens que furent introduits Rattus rattus L., Rattus norvegicul Berke., et la souris Mus musculus L.

Les mythes tendent donc à prouver que le rat est introduit dans les îles avant le kava puisque lorsqu'il le découvre la plante est inconnue de l'homme. Ou encore qu'il s'agit d'une plante non cultivée («... le vrai kava pour remplacer le faux...") et donc provenant de lá forêt où elle vit à l'état sauvage et que l'homme aurait trouvée grâce au rat. Le phénomène pourrait s'être produit dans une île où le mythe aurait pris naissance puis le mythe aurait voyagé avec la plante au gré des migrations et des échanges de matériel végétal.

Si l'étude de la mythologie ne permet pas de conclusions précises, il n'en est pas moins vrai qu'elle indique une origine commune, qu'elle souligne la contribution du rat dans la découverte de la plante sauvage et qu'elle montre que l'introduction du kava dans chaque île paraît ancienne puisque chaque culture se l'approprie en oubliant qu'elle fut introduite (à l'exception de Tanna qui en accepte la réalité).

\section{Le Piper methysticum Forst. F. eSt UN kAVA STÉRILE}

La cérémonie du kava fut observée pour la première fois par Le Maire et Schouten en 1616 à Futuna (Brosses, 1756). Solander et Parkinson furent très certainement les premiers scientifiques à s'intéresser à la plante dont ils décrivent les usages et les modes de préparation à Tahiti et dont ils font le premier croquis (Parkinson, 1773). La première description du kava est due à J. G. A. Forster, qui lui donna le nom de Piper methysticum, ou poivre enivrant, Methysticum étant la transcription latine du grec Methustikos dérivé de Methu qui signifi- rait "boisson enivrante" (Steinmetz, 1960 ; Lebot et Cabalion, 1986). Mais cette première description que l'on trouve dans «Supplementum plantarum, Emendanda " (Forster, 1781) n'est pas reconnue comme valable par le code international de nomenclature pour la simple raison que les échantillons récoltés par les Forster appartenaient en fait à deux espèces : $P$. methysticum et Macropiper latifolium. La description du premier binôme par le jeune Linné en 1781 fut considérée fausse par son auteur à la date de sa publication (Smith, 1981). Piper methysticum L. f. (1781) n'est donc pas reconnu par le code de nomenclature parce qu'il s'agit en réalité d'un synonyme de Macropiper latifolium (L.f.) Miq. Lorsque Miquel (1840) publie une première description du genre Macropiper, il ne manque pas de préciser la mention suivante : "Piper latifolium L.f., Suppl. Pl. Emendanda : 468 (1781); non P. methysticum L. f., op. cit. : 91 , nomen auctori non gratum ". La description qui fait date est donc celle que J. G. A. Forster publia dans sa thèse de doctorat ( De Plantis Esculentis Insularum Oceani Australis ", 1786) :

«P. foliis cordatis acuminatis multinervis : spicis axillaribus folitariis brevissimis, pedunculatis. patentissimis. F.

Species caute diflingnenda a Piper latifolio quod in Supplem. Plantar. p. 91. nescio quo casu Piper methysticum vocatur. Etinim non solum notis botanicis plurimis a vero Pipere methystico, latifolium illud discrepat, sed etiam toxica qualitate caret. neque in hune ufum ab incolis unquam adhibetur. sponteque nafcit per omnes fere insulas oceani australis intra tropicos fitas.

Piper methysticum verum inter plantas cultas earundem insularum passim reperitur, iis tamen exceptis, quae nigrae gentis sedes sunt. Novis Hebridibus et Caledonia Nova."

Les synonymes botaniques sont nombreux, on trouve Macropiper methysticum Miquel, Piper decumanum Opitz, Piper inebrians Solander ex Parkinson et Bertero et Piper spurium Forst. Les espèces apparentées sont endémiques à la Mélanésie, il s'agit de Piper gibbilimhum C.DC., P. plagiophyllum K. Schum. \& Lauterb. et de P. vichmannii C.DC.

A notre connaissance, il est impossible de trouver le $P$. methysticum à l'état spontané. Le kava, tel qu'il est décrit par les botanistes et tel qu'il est représenté dans les plus grands herbiers, correspond toujours à un individu cultivé. La présence du kava dans une île résulte toujours de l'intervention de l'homme qui le multiplie exclusivement par bouturage. Lorsque le kava n'est plus cultivé. il disparaît car il est inapte à se reproduire par voie sexuée. 
Les horticulteurs ne connaissent pas les sexes de leurs cultivars, ne font aucune différence entre mâles et femelles. De Lessert (1837) est certainement le premier botaniste à avoir signalé que l'espèce était diö̈que. Cuzent (1857) dit rapidement que "les fruits sont monospermes ", et Barrau (1957) précise que "le fruit est une baie à une graine ". Ces remarques sur le fruit du kava sont étonnantes car, pour notre part, et malgré nos nombreuses prospections, nous n'avons jamais observé une seule fructification. De mémoire d'horticulteurs également, on n'en a jamais vu. Parmi toutes les références bibliographiques traitant du kava, deux seulement soutiennent le contraire en donnant la description de graines mais sans renvoyer à un échantillon d'herbier (Cuzent, 1857 et Barrau, 1957). Après vérification, le seul échantillon d'herbier cité comme un kava et portant des fruits (I. et Z. Baker, n" 15, 2/11/38, Santo, British Museum) est en fait un "faux kava": Macripiper latifolium Forst. (Lebot et Cabalion, 1986 ; comm. pers. de P. S. Green).

De Lessert (1837) représente des inflorescences mâles mais aucune fructification, de même que Degener en 1940. Ce dernier précise qu'il n'a pu en observer dans aucune des plantations qu'il a visitées à Hawaï et signale que Hillebrand, un récolteur infatigable, n’a pu les représenter dans sa flore, plusieurs années auparavant, car elles étaient introuvables. Enfin. Hänsel (1968) confirme ces informations. Tous ces auteurs affirment ne pas avoir observé d'inflorescences femelles. Les identifications imprécises sont fréquentes dans le genre Piper, il est donc difficile de déterminer ce qui relève de mauvaises observations ou de l'absence réelle de fleurs femelles en ce qui concerne les cas cités dans la littérature puisqu'il nous est arrivé d'en trouver au cours de nos prospections. Les plants des deux sexes ne peuvent cependant, être différenciés par leurs caractères végétatifs. Les fleurs sont très petites et l'observation des étamines par exemple ne peut se faire qu'à maturité. S'il est vrai que les individus femelles sont effectivement rares (moins de un plant sur cinq environ), ils existent néanmoins en nombre suffisant pour permettre d'éventuelles fécondations. Il n'existe à notre connaissance aucune différence d'ordre morphologique entre individus mâles et femelles, si ce n'est la longueur de l'inflorescence. La floraison est de toute façon peu abondante et il nous est arrivé de rencontrer des horticulteurs qui n`avaient jamais observé les fleurs de leurs kava, c'est dire le peu d'importance que présente ce caractère pour les insulaires.

Ces observations réalisées sur le terrain sont confirmées sur les échantillons de Piper methysticum Forst. f. existant dans les plus grands Herbiers du monde. En 1986 nous avons contacté ou visité les Herbiers suivants : Kew, Londres, Paris. Genève. La Haye, Kuala Lumpur, Singapour, Bogor, Sydney, Melbourne. Brisbane, Nouméa, Auckland, Christchruch, Lae, Guam, Honolulu. Missouri et Harvard. Aucun des échantillons de $P$. methysticum existant dans ces Herbiers ne porte de fruit et les individus femelles sont toujours très rares. Les botanistes sont unanimes pour dire que les inflorescences femelles sont difficiles à observer mais ne se prononcent pas sur l'origine du phénomène.

En culture, les inflorescences se développent puis tombent avant datteindre leur maturité. Le Piper methysticum Forst. étant une espèce dioïque, le mode de reproduction sexuelle devrait se faire par allopollinisation obligatoire. Le pollen semble être produit en quantité suffisante par les inflorescences mâles. Il est de type collant et globuleux et ne paraît pas pouvoir être véhiculé par le vent, à la rigueur par l'eau de pluie. Nous pensons que la pollinisation pourrait être entomophile plutôt qu'anémophile. Lorsque des pollinisations sont effectuées manuellement, les fleurs femelles situées à la base de l'inflorescence paraîssent être fécondées et de jeunes baies commencent à se développer tandis que les fleurs situées au sommet de cette inflorescence ne présentent aucune réaction à cette pollinisation. Avant que les baies natteignent leur maturité le pédoncule se détache de son point d'insertion sur le rameau et l'inflorescence tombe. Si l'on procède à une dissection de la jeune baie à ce moment-là on observe que l'ovaire contient une graine à albumen immature. L'échec de ces pollinisations montre que le transport du pollen n'intervient pas dans le phénomène de la stérilité chez le $P$. methysticum.

On constate une remarquable constance du nombre chromosomique de base chez Piper qui est de $\mathrm{x}=13$ (Samuel, 1986). Nous avons effectué des comptages sur pointes racinaires d'un cultivar (ni kava pia) de Piper methysticum originaire de Vanuatu. Le cultivar étudié est un decaploïde $\dot{a} 2 \mathrm{n}=130(=10 \mathrm{x})$ chromosomes (Lebot, 1988). C'est la première fois que ce niveau de ploïdie est observé pour un Piper. Ce très haut niveau de ploïdie pourrait expliquer en partie le phénomène de la stérilité du Piper methysticum. Ce decaploïde est donc très certainement un mutant somatique d'un pentaploïde à $2 \mathrm{n}=65(=5 \mathrm{x})$ chromosomes. En effet, chez les espèces de Piper cultivées et multipliées par voie végéta- 
tive ( $P$. betle, $P$. longum, $P$. nigrum), les doublements successifs du nombre de chromosomes sont fréquemment observés et résulteraient d'endomitoses.

\section{Le Piper wichmanni C. DC. est l'ancêtre} DU KAVA.

Au cours de nos prospections à Vanuatu, les horticulteurs nous ont présenté le seul kava que l'on puisse parfois trouver à l'état spontané et qu'ils appellent en bichelamar le "kava sauvage " (wael kava). Ce kava est, d'après la tradition orale du nord de l'archipel de Vanuatu, le premier kava, c'est-à-dire celui qu'utilisaient les ancêtres et dont proviennent tous les cultivars utilisés aujourd'hui. Bien que la morphologie de cette plante soit très proche de celle du Piper methysticum, son inflorescence est plus longue que celle des cultivars traditionnellement utilisés, aussi longue que la nervure centrale du limbe. Ce kava sauvage n'est que très rarement consommé, sauf les jours de fête pour couper les "grands crus", lorsque les volumes disponibles sont insuffisants. Nous avons pu l'identifier à Piper vichmanii C. DC. tel qu'il est décrit par Saüer et Hänsel (1967) et Chew (1972), (Lebot, Cabalion et Lévesque, 1986). L'un de nos collègues (Cabalion, comm. pers., 1985) devait judicieusement observer que l'échantillon de racines analysé par Saüer et Hänsel avait été identifié cinq ans plus tard comme étant $P$. wichmannii par Chew. Cette espèce était donc la seule du genre Piper, avec le $P$. methysticum, à posséder des kavalactones, les principes actifs qui font du kava une plante hors du commun.

Nous avons donc pris soin d'envoyer à Chew des échantillons provenant de $P$. methysticum et de $P$. wichmannii cultivés à la Station d’Agriculture de Tagabé afin d'obtenir une confirmation des premières identifications. Ses commentaires furent les suivants :

.... From the point of view of formal taxonomy, I am not inclined, however, to actually put the two species into one. This is largely because $P$. methisticum is known only from gardens (not in the wild) and should really not be considered as a "species ", but as a putative cultivar. The kara is such an important economic " species " that to consider it as including Piper lichmamnii would certainly cause conceptual and practical problems both for taxonomy and " agro-botany ". It is a different matter of course, if Piper methysticum should be discovered in the forest".

(communication personnelle du 6 mars 1986)

Il semble donc que si les horticulteurs océaniens ne font aucune différence entre ces deux espèces, il en va de même pour les spécialistes qui considèrent finalement que l'une rassemble les formes sauvages de kava (P. wichmannii) et l'autre les formes cultivées ( $P$. methysticum). Il est intéressant de constater que ce problème n'est pas nouveau. En 1874 déjà, l'ethnologue russe $\mathrm{N}$. de Mickloucho-Maclay travaillant sur la côte nord-ouest de la Nouvelle-Guinée, qui porte aujourd'hui son nom, écrivait :

"Finding that the botanical character of the leaves of the " keu " plants taken from different bundles, presented some differences. I sent the whole concern (bundles, flattened leaves, etc.), to Dr. Scheffer for complete examination, with the request to tell me by-and-by his opinion: whether all the "keu" specimens belonged to the same species (Piper methisticum) or not. The same forenoon I received a short note from Dr. Scheffer, written in haste in the Botanical Garden, with the statement that the bundles of " keu " contained two different species of Piper and both different from the Piper methysticum, but that through the absence of flowers and fruits it was impossible for him to determine the species.

... I had the opportunity of stating the information from the Rev. W. G. Lawes, that the Piper methysticum grows wild on the south-east of New-Guinea without the natives knowing or making use of it. Rev. G. Brown wrote to me a few days ago about a similar case in New Britain and New Ireland, and the Solomons islands, where Piper methysticum (or an allied species) grows wild, but the natives don't know the use of it (I got large roots of it, and natives from other islands said it was the true "kava", but not being cultivated it was coarse.) ".

Le Piper wichmannii C. DC. a été décrit pour la première fois en 1910 par De Candolle (Nova Guinea Bot. 8 (2) : 418 . NouvelleGuinée Néerlandaise, côte du nord : Atasrip $\mathrm{n}^{\circ}$ 39 Exp. Wichmann, 1903). Cette espèce arbustive de sous-bois est dioïque, les inflorescences femelles sont invariablement plus courtes que les mâles et les pédoncules plus petits que les pétioles, comme chez $P$. methysticum. Les fleurs mêles ont deux étamines et les fleurs femelles trois stigmates soudés, des bractées rondes et peltées. Les fruits sont sessiles, de forme conique et très nombreux. Cependant, si une inflorescence femelle peut porter plusieurs centaines de ces baies à une graine, il semble que le pourcentage de germination soit très faible. Un essai que nous avons réalisé avec plusieurs centaines de ces graines récoltées sur rîle de Karkar, sur la côte nord de la PapouasieNouvelle-Guinée, n'a donné aucun résultat après semis. Les $P$. wichmannii sont des arbustes que l'on trouve toujours isolés en sous-bois et il est très rare de rencontrer deux plants à proximité l'un de l'autre. Lorsque les inflorescences sont à maturité, les baies sont d'un 
orange vif, très attirant pour les chauve-souris que nous pensons être responsables de la dispersion de ces fruits.

Sur l'île de Baluan (Amirauté), en PapouasieNouvelle-Guinée, le $P$. wichmannii est cultivé avec deux autres cultivars de $P$. methysticum et tous trois sont exclusivement multipliés par voie végétative. Pour les habitants de cette île, tous trois sont des kava qu'ils nomment par le nom générique : Kau. Kau pel et Kau pwusi sont des cultivars de $P$. methysticum et Kau kupve est une forme cultivée de $P$. wichmannii. Les horticulteurs de Baluan nous ont affirmé que leurs cultivars étaient tous stériles et ne produisaient jamais de graines (y compris la forme cultivée de $P$. wichmannii). Après examen plus poussé de la situation, il s'est avéré que tous les individus présents sur cette île d'environ $5 \mathrm{~km}^{2}$, étaient des mâles. Dans quelques îles de Vanuatu, certains individus de $P$. wichmannii poussent à l'état spontané d'après les habitants, mais personne ne se souvient avoir vu un jeune plant issu de graine. Lorsqu'on les rencontre en forêt, il s'agit en réalité de plants déjà âgés, épargnés dans le cycle des systèmes de culture à longue révolution, ou mis en terre accidentellement.

Pour Chew (1972), il s'agirait peut-être de l'espèce de Piper la plus fréquente en PapouasieNouvelle-Guinée. Nous ne partageons son avis qu'en partie car nos prospections dans ce pays nous ont montré que $P$. gibbilimbum ou $P$. plagiophyllum sont beaucoup mieux établis dans les régions des hauts plateaux tandis que $P$. wichmannii se plaît de 0 à $1000 \mathrm{~m}$ d'altitude sur la grande terre et dans les îles environnantes. Par contre il s'agit incontestablement de l'espèce de Piper la mieux installée aux îles Salomon. Nous sommes cependant d'accord avec lui pour reconnaître que cet espèce est la plus distinctive du genre, avec bien sûr, $P$. methysticum, en raison de son port érigé et de ses tiges orthotropes issues d'un collet basal. Lorsque des individus appartenant à ces espèces sont plantés à partir de boutures, les différences observées ne sont pas plus significatives que celles rencontrées entre plusieurs cultivars, elles concernent les caractères morphologiques et non pas la croissance des plants. Les différences essentielles sont relatives au système racinaire plutôt qu'à l'appareil végétatif aérien. Les racines de $P$. wichmannii sont beaucoup plus lignifiées que celles des cultivars de $P$. methysticum. La souche et les racines contiennent de l'amidon en moindre proportion et un rendement en matière sèche beaucoup plus faible. Comme nous le verrons plus loin, les caractéristiques chimiques sont elles aussi sensiblement différentes. En réalité le $P$. wichmannii présente des caractères sauvages et le $P$. methysticum des caractères améliorés résultant d'un processus de domestication et de sélection. De plus, les récents travaux que nous avons entrepris montrent que namaloku kau, une forme cultivée de $P$. wichmannii, est un pentaploïde à 65 chromosomes. La tradition orale qui précise que le Kava des ancêtres est aussi lancêtre du Kava, semble donc être confirmée par les données génétiques.

En ce qui concerne $P$. wichmannii, les cent onze échantillons existants dans les Herbiers sont tous originaires de Papouasie-NouvelleGuinée, des Iles Salomon ou de Vanuatu. Cette espèce n'existe pas en Micronésie ni en Polynésie et elle ne semble pas avoir été récoltée dans le sud-est Asiatique ou en Indonésie. Les Herbiers de Kuala Lumpur, Singapour et de Bogor n'en possèdent aucun échantillon, il s'agirait donc d'une espèce endémique à l'arc Mélanésien et dont la dispersion se limiterait à cette région. C'est d'ailleurs ce que rapporte Chew (1972) en précisant que son aire de distribution s'étend exclusivement de la Papouasie à l'archipel Bismarck et aux îles Salomon.

Il semble que le premier échantillon de kava récolté sur l'île de Tanna, au sud de l'archipel de Vanuatu, était un cultivar et non pas une espèce poussant à l'état sauvage : "Piper methysticum verum inter plantas cultas earundem insularum passim reperitur,..." (Forster, 1786). En 1910, quand C. De Candolle publie sa Flore "Nova Guinea ", la description qu'il donne de $P$. methysticum est la suivante «Espèce cultivée dans la plupart des îles du Pacifique " (p. 415). C'est d'ailleurs la seule fois, dans le chapître consacré au genre Piper, où il estime nécessaire de mentionner le caractère "cultivée " de cette espèce. À la page 418 , du même ouvrage, il donne une description du $P$. wichmannii C. DC. qui ne diffère en rien de celle du $P$. methysticum et ne donne pas de descriptions des fruits ni des graines (De Candolle, 1910, Nova Guinea $8: 415$ et 418 ). C'est très certainement pour cette raison que Chew (comm. pers., 1987) ne peut observer de différences morphologiques entre ces deux plantes, de même que les horticulteurs de Vanuatu, des Iles Salomon ou de Baluan observent des différences morphologiques entre des formes, cultivées ou sauvages, appartenant à l'un ou à l'autre de ces deux binômes mais qui sont du niveau intraspécifique, c'est-à-dire qu'elles concernent des cultivars. S'il y a un domaine où ils excellent c'est bien celui de la différentiation des caractères, mais ils savent aussi quand cette différentiation atteint le niveau interspécifique. 
En accord donc avec les spécialistes et les hommes de terrain, nous concluons que le $P$. methysticum ne devrait pas être considéré comme une espèce, puisqu'il est stérile, ni une variété, puisqu'il semble rassembler plusieurs génotypes mais tout simplement comme un ensemble de cultivars stériles. Pour les raisons évoquées par Chew, c'est en fait le $P$. wichmannii qui devrait disparaître pour intégrer le premier binôme de J. G. A. Forster (1786). Cependant, le fait que Chew lui même refuse de confondre ces deux binômes nous incite à traiter $P$. methysticum comme une espèce.

\section{LES CULTIVARS SONT DES MORPHOTYPES.}

Nous avons vu à plusieurs reprises qu'il n'existaient pas un kava mais plusieurs. Il est d'ailleurs pratiquement impossible de parler du kava au singulier avec les horticulteurs océaniens car il leur paraît essentiel, sinon fondamental, d'orienter la discussion vers les différentes caractéristiques de leurs kava. Ils tiennent à préciser, dès que possible, de quel "cépage " il s'agit. Comme si ce qui faisait la valeur de cette plante était justement sa variabilité et les spécificités de l'usage et des différents variants.

On appelle variété une subdivision de niveau inférieur à l'espèce et correspondant à un génotype, c'est-à-dire à l'ensemble des gènes que contient et transmet un individu. Il semble néanmoins que ce terme, très imprécis, soit trop souvent utilisé et parfois à tort ou à travers. Les kava par exemple, sont multipliés par l'homme par voie végétative. Une population issue d'un individu par multiplication végétative forme un clone et comporte un seul génotype, il s'agit donc logiquement d'une seule variété. Tous les clones de kava résultent, comme nous l'avons vu, d'un processus de sélection traditionnelle. Or une variété de plante cultivée issue d'une sélection s'appelle un cultivar.

Le cultivar, tel qu'il est défini par le Code International pour les Plantes Cultivées (Bradenburg, 1985) est "un ensemble de flantes qui sont clairement différenciées par des caractères morphologiques, physiologiques, cytologiques, chimiques ou autres, et qui les conservent après reproduction sexués ou asexuée ". Il s'agit là, malheureusement, d'une définition très générale et qui concerne une grande diversité de formes cultivées.

La découverte de nouveaux cultivars par les horticulteurs continue à se faire. Les enquêtes nous ont appris que plusieurs dénominations sont récentes. La procédure est souvent la même dans toutes les îles: un horticulteur plus observateur que les autres constate que certains caractères permettent de différencier un plant d'un autre et clone, à partir du pied-mère, le nouveau cultivar. Celui-ci est baptisé en langue vernaculaire selon le caractère dominant, ou parfois même tout simplement du nom du sélectionneur. Il existe au sein du clone une différenciation génétique responsable de la modification de certains caractères morphologiques. Les mutations concernent les cellules somatiques, c'est-à-dire non sexuelles, et produisent des chimères ou des individus porteurs de tissus génétiquement différents (Silander, 1985). Ce phénomène n'est pas particulier au kava, chez les plupart des végétaux multipliés exclusivement par voie végétative, des variations peuvent se produire. Cette hypothèse d'un polymorphisme d'origine asexuée coïnciderait bien, nous semble-t-il, avec les observations, sur le terrain. Il nous est arrivé fréquemment au cours de nos enquêtes d'entendre les horticulteurs affirmer que certains de leurs cultivars "changeaient" lorsqu'ils les multipliaient.

Pratiquement, les horticulteurs opèrent ainsi :

Les jardins traditionnels comprennent environ 10 à 20 cultivars différents, cultivés en association avec d'autres espèces. Un plant peut présenter un développement végétatif normal sans que son apparence permette de préjuger de la qualité du breuvage qu'il procurera. L'horticulteur va donc le déraciner et observer dans un premier temps le rendement du plant, c'est-à-dire le poids total de racines consommables. Il va ensuite récupérer toutes les boutures de tiges consommables et les laisser " en attente ", enfouies dans le trou laissé par l'arrachage du plant. Le soir même, ou dans les jours qui suivent, il va consommer le kava avec des confrères, jamais seul. C'est à ce moment-là qu'il évalue les qualités du plant. Le kava obtenu peut être fort, agréable, calmer ses rhumatismes ou bien l'empêcher de dormir... Si les conclusions de lexpérience sont intéressantes, les boutures "en attente " seront soigneusement récupérées, taillées et habillées, puis mises en terre où il convient. Si le kava est désagréable, les boutures seront tout simplement oubliées dans ce trou de terre déjà épuisée et seront rapidement envahies par les adventices. Autrement dit, l'horticulteur sélectionne et clone ses plants en fonction de deux critères : le rendement total et la qualité du breuvage. Cette sélection se fait traditionnellement à chaque récolte de plant et les horticulteurs sont experts en la matière. Parce que cette plante fait partie de leur patrimoine culturel, ils 


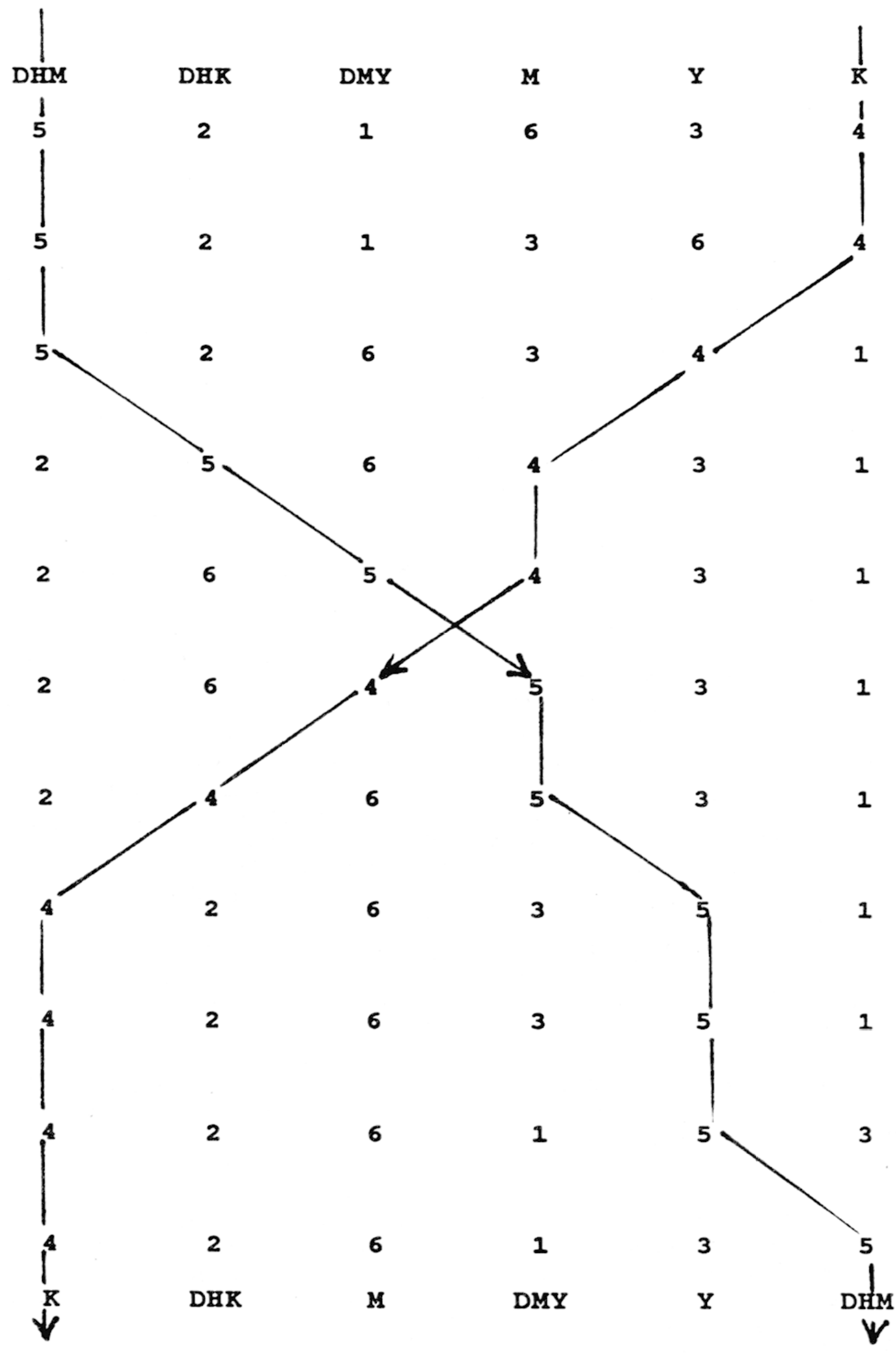

Fig. 1. -- Généalogie des chimiotypes conduisant d'une forme de $P$. wichmamii très rarement consommée (52I634). a un cultivar de $P$. methysticam très apprécié (426135). La sélection clonale des chimiotypes à chaque récolte de plant vise à une amélioration de leffet ressenti par le buveur, en réduisant la proportion de dihydrométhysticine (5) et an augmentant la proportion de kawaïne (4). 
entretiennent leurs collections de cultivars avec soin.

On n'accorde pas à cette plante de soins aussi minutieux qu'aux ignames et aux taros, mais à chaque cultivar correspond une technique de culture en fonction de son rang dans la classification locale. Les cultivars de kava font l'objet de classifications dont les principes et les critères diffèrent d'une île à l'autre. Ces cultivars sont classés hiérarchiquement selon leur importance dans les relations deechanges, c'est-à-dire suivant leur utilisation sociale et rituelle.

Cette classification concerne l'ensemble de ce que les horticulteurs appellent les "différentes sortes de kava", observées sur le terrain et que nous avons comparées entre elles au cours de nos enquêtes. Ces formes végétales locales sont des clones. Un même clone, donc un même génotype, peut présenter selon les circonstances des phénotypes différents. Classer des clones, c'est étudier l'homogénéité d'une population issue par multiplication végétative (par bouturage) d'un seul individu sélectionné, reproduit à $\mathrm{n}$ exemplaires génétiquement identiques, à la mutation somatique près. L'objectif de la classification traditionnelle est donc d'observer la variabilité interclonale mais aussi intraclonale. lorsqu'elle existe. Cette classification révèle par la minutie de ses observations, une science du kava très sophistiquée que seuls les anciens pratiquent, mais qu'aujourd'hui encore quelques jeunes continuent à apprendre en observant les caractères de chaque forme cultivée. Certaines techniques et cultivars sont endémiques à certaines îles, ou même à certaines régions, et lorsqu'on les retrouve ailleurs que sur leur aire d'origine, souvent à la suite d'offrandes coutumières, ils sont considérés par lcs populations locales comme étrangers à leur terroir. Les boutures circulent très facilement à l'occasion d'échanges traditionnels mais les cultivars présentent un ensemble de caractères morphologiques significatifs seulement pour les horticulteurs d'une île. Ils sont valables pour l'identification des cultivars locaux, mais ne se prêtent pas à la reconnaissance de ces mêmes cultivars par la population d'une autre île.

Ces caractères se rapportent essentiellement à l'appareil végétatif et sont sujets à de grandes variations en fonction de l'environnement. Bien qu'utilisés traditionnellement, l'efficacité de ces systèmes locaux de discrimination des cultivars est réduite, dès lors qu'ils s'exercent en dehors du milieu d'origine. Les descriptions obtenues au cours des enquêtes ethnobotaniques ne sont significatives que pour l'aire de culture concernée. De même, un certain cultivar peut être classé différemment dans les hiérarchies de deux îles différentes. Ces formes végétales locales correspondent donc à des morphotypes. Il s'agit essentiellement de cultivars obtenus au hasard ou par sélection généalogique. Les kava peuvent dont être comparés à des cépages puisque d'après le dictionnaire d'agriculture (A.C.C.T., 1977) il s'agirait de "population de clones suffisamment apparentés pour être pratiquement identifiables soit par un caractère morphologique, soit par un caractère végétatif ou cultural $»$.

Les cultivars de kava sont dispersés géographiquement sur les îles d'Océanie qui comportent une mosaïque de biotopes où ils ont évolué. Il est très probable que certains d'entreeux résultent de mutations somatiques. Par comparaison aux résultats obtenus par reproduction sexuée, la reproduction asexuée devrait ralentir le processus d'évolution et limiter la variabilité puisqu'il est communément admis que le clone est un réplicant du génotype. Certains auteurs (Silander, 1985; Cook, 1985) suggèrent plutôt que la multiplication végétative contribue très efficacement au polymorphisme. Dans le cas du kava, espèce à reproduction exclusivement végétative, la variabilité des caractères morphologiques est grande mais pas surprenante. En effet si l'on compare le kava à d'autres espèces multipliées exclusivement par voir végétative, cette variabilité semble normale. Walter (comm. pers., 1988) a recensé dans la région du centre de l'île de Pentecôte, à Vanuatu, tous les cultivars traditionnellement utilisés par la population de langue Apma qui compte 4304 locuteurs. Le nombre de cultivars différenciés et utilisés par les habitants de cette région de $120 \mathrm{~km}^{2}$ seulement, est pour chaque espèce :

$\begin{array}{lc}\text { Colocasia esculenta } & 101 \text { cultivars } \\ \text { Dioscorca spp. } & 90 " \\ \text { Artocarpus altilis } & 42 " \\ \text { Musa spp. } & 25 " \\ \text { Abelmoschus manihot } & 18^{\prime \prime} \\ \text { Piper methysticum } & 14^{\prime \prime} \\ \text { Ipomoea hatatas } & 13^{\prime \prime} \\ \text { Pandanus spp. } & 11^{\prime \prime} \\ \text { Manihot utilissima } & 9 " \\ \text { Xanthosoma sagittaefolium } & 2 "\end{array}$

On observe que ce sont les espèces les plus anciennement introduites qui présentent le plus de cultivars, tandis que les espèces d'introduction récente ( 1 à 2 siècles) en ont peu. Cette observation est aussi valable pour les autres îles du Pacifique. D'une manière générale, on compte moins de cultivars en Polynésie qu'en Mélanésie pour une espèce donnée, tout simplement parce que les cultures y sont plus 
récentes. A cette ancienneté de la multiplication et de la sélection doit s'ajouter la diversité génétique existante au départ et qui est beaucoup plus grande pour les espèces originaires d'Indo-Océanie plutôt que d'Amérique (Ipomoea batatas, Xanthosoma sagittaefolium). Larsen (1985) de son côté, a recensé 74 cultivars de patate douce, 42 cultivars de Manioc $(M$. utilissima) et 42 cultivars de Cocoyam ( $X$. sagittaefolium) pour l'ensemble de l'archipel de Vanuatu. Le kava est donc peu polymorphe si on le compare aux autres cultures traditionnelles. Se pose cependant la question de définir précisément la nature de ces "variétés horticoles " (Barrau, 1962) car le terme "cultivar" est, comme nous venons de le voir très approximatif. Notre expérience du problème nous a appris que l'appelation qui convient dans le cas présent est celle de morphotype. En effet, on nomme morphotype, un phénotype dont les caractères morphologiques ont été décrits et codés de manière à le distinguer des autres. Ce qui revient à reproduire ce que font traditionnellement les horticulteurs océaniens. La multiplication asexuée semble donc être responsable du polylmorphisme observé chez ces cultures traditionnelles. Barrau (1957) précise «... que les variétés horticoles d'arbre à pain sont surtout nombreuses dans les îles où dominent des formes parthénocarpiques multipliées par voie végétative». Cette variabilité est donc morphotypique plutôt que génotypique.

Bien que les nomenclatures insulaires présentent des similitudes dans leur organisation, il est plus aisé de les aborder séparément. De tous les pays d'Océanie où l'on cultive le kava, Vanuatu est incontestablement celui qui conserve la tradition la plus riche. Les cérémoniaux, les modes de culture ainsi que la classification des cultivars sont à l'image de l'archipel, d'une diversité extrême. Chaque île sélectionne les siens et s'y identifie. Le nom vernaculaire rappelle le caractère principal du cultivar, une légende ou plus simplement le nom de celui qui, le premier, sélectionna le clone. La description souvent sommaire donnée par l'horticulteur, et la signification des noms vernaculaires permettent d'établir des hypothèses de concordance dont seule l'utilisation de descripteurs botaniques objectifs peut confirmer l'éventuelle validité.

\section{Fidji :}

Les cultivars utilisés par les horticulteurs aujourd'hui à Fidji sont au nombre de 12 ; Les cultivars Kabra, honolulu et business sont d'après les horticulteurs d'introduction récente et n'ont pu être retrouvés dans la littérature. Seemann recense seulement six cultivars en 1865 , Steinmetz (1960) ne rapporte que cinq descriptions et noms vernaculaires de cultivars à Fidji et Parham (1964) précise que selon lui il n'existe plus que cinq cultivars à Fiji à cette époque
("... but up to the présent only five have been found, two of which are not very common " $p$. 3). Er. 1984 enfin, Heinlein et al. récoltent cinq cultivars seulement malgré leur présence permanente dans ce pays. Sur les trois principales îles de l'archipel de Fidji, Viti Levu, Vanua Levu at Taveuni, les horticulteurs connaissent 16 dénominations différentes. D'après les enquêtes menées dans ces îles, les autres régions de l'archipel utiliseraient les mêmes cultivars. Sauf probablement Rotuma qui est une colonie Polynésienne. Les dénominations fidjiennes suivent un principe très simple de différentiation morphologique. Le nom du cultivar tend à résumer son apparence en suivant une clé de détermination dichotomique. Les cultivars sont ou "clairs", Vula, ou "sombres", Loa. Ils sont ensuite différenciés selon la conformation de leurs entrenœuds qui peuvent être "longs", Balavu ou "courts", Leka ou encore "longs et gros comme un pieu, "Dokobana" (entrenœuds se disant Kasa) :

Loa Kasa Leka: Sombre avec de courts entrenœuds.

Loa Kasa Balavu: Sombre avec de longs entrenœuds.

Vula Kasa Leka: Clair avec de courts entrenœuds.

Vula Kasa Balabu: Clair avec de longs entrenœuds.

Dokobana Loa: Sombre avec des entrenœuds longs et épais.

Dokohana Vula: Clair avec des entrenœuds longs et épais.

Qila Ealavu : Qui provient du lieu-dit "Qila», sur lîle de Taveuni, avec de longs entrenœuds.

Qila Leka : Qui provient du lieu-dit «Qila », avec de courts entrenœuds.

Malakaro: Signifie "tacheté".

Matakaro Balavu: Tacheté, avec de longs entrenœuds.

Matakaro Leka: Tacheté, avec de courts entrenœuds.

Loa : Sombre.

Damı : Rouge.

Kabra : Nom d'un horticulteur.

Honolulu : Introduction récente dont l'origine géographique est douteuse.

Business : Introduction récente.

D'après les horticulteurs, les cultivars "clairs " sont réputés fournir le meilleur kava, mais sont beaucoup moins précoces et résistants aux maladies que les cultivars "sombres".

\section{Polynésie :}

Dans cette vaste région seules les îles Tonga, Samoas, Wallis et Futuna pratiquent encore la culture du kava. Aux îles Cook, à Niue, aux îles de la Société, aux îles Marquises et aux Hawaï, le kava n'est plus cultivé et tend à disparaître complètement. A Tonga, le procédé de dénomination employé pour différencier les sept cultivars traditionnellement utilisés est 
similaire à celui de Fidji. Par exemple, Akau signifie "long" et Huli signifie "sombre", d'où le nom de Akau Huli pour un cultivar sombre dont les entrenœuds sont longs. A Tonga, la culture du kava est quelquefois difficile et du fait de la consommation croissante les commerçants en importent régulièrement des Iles Samoa. A Wallis et Futuna (trois cultivars), aux Samoas (sept), c'est aussi la morphologie du plant qui détermine le plus souvent le nom du cultivar. Les classifications sont néanmoins rudimentaires en raison du très faible nombre de cultivars utilisés dans ces pays. A Wallis, le kava n'est guère consommé que lors des cérémonies coutumières mais tous les ménages le cultivent pour répondre justement à leurs obligations sociales. A Futuna, le kava est consommé quotidiennement sous forme de racines fraîches, la culture est destinée à la consommation locale mais se fait pourtant en plantations importantes. Le climat et les sols de Futuna sont très favorables à sa culture.

A Tahiti, le pharmacien de Marine Cuzent répertoriait en 1856,14 cultivars de $A v a$ dont les caractéristiques concernaient essentiellement la qualité enivrante des racines, la hauteur des plants, la grosscur des tiges, la longueur des entrenœuds et enfin la couleur des feuilles. Le kava n'est plus cultivé depuis les interdits imposés par les missions à la fin du XVIII siècle. Curieusement, la situation n'a guère changé depuis 1927, époque à laquelle Lewin écrivait :

"Actuellement et depuis de nombreuses années, la culture a iout à fait cessé à Tahiti. Aux environs de 1830 déjà, il n'était plus possible de se procurer un seul exemplaire de la plante et beaucoup d'indigènes ne la connaissent même plus de nom. On a trouvé à l'intérieur de Tahiti quelques rares exemplaires disséminés, rabougris et minces de tiges. Il en va de même à Kaiatéa et à Moorea ». (Phantastica, p. 233)

A notrc connaissance, il ne reste que quelques souches apparemment entretenues dans la moyenne vallée de la Fataua (environ $300 \mathrm{~m}$. alt.) et de la Papenoo. Brown avait recensé 21 cultivars aux îles Marquises en 1935, aujourd'hui le kava a complètement disparu des îles de Nuku Hiva, Ua Pohou, Hiva Hoa, Tahuata et de Mohotane. Les vallées de toutes ces îles ont été visitées et prospectées sans qu'il soit possible de rencontrer un seul kava. D'après les habitants les derniers survivants auraient disparu il $\mathrm{y}$ a près de 30 ans à la suite des déracinages successifs opérés par les cochons sauvages. I! reste quelques plants à Ua Huka et une petite population à Omoa dans la haute vallée de Hanavave, à Fatu Hiva, en bordure du chemin qui conduit de Omoa à Ouia.

À Hawaï, en 1933, on distinguait encore sept cultivars (Lebot et Cabalion, 1986). Le kava n'est plus cultivé à Hawaï mais il s'est remarquablement adapté à l'environnement du côté au vent de l'île de Oahu (nord-est). Les botanistes locaux disent qu'il est désormais «naturalized " bien qu'à mon avis ce terme soit impropre pour une espèce incapable de se reproduire par voie sexuée. On peut encore y trouver quelques populations le long des ruisseaux ombragés et encaissés des fonds de vallées. Il semble qu'il ait conservé cependant un certain rôle dans ce qu'il reste de la pharmacopée traditionnelle polynésienne car les plants observés présentaient des signes d'entretien et de désherbage autour des souches. Si le kava n'est plus cultivé, les derniers plants sont donc préservés par une partie de la population. Nous avons pu récolter 10 cultivars dans les vallées de Oahu mais un seul sur l'île de Hawaï, dans le district de Puna. Cependant les discussions que nous avons eues avec plusieurs botanistes ayant parcouru les vallées de l'archipel confirment la présence de population isolées dans les vallées des autres îles (Maui, Kauai).

\section{Papouasie, Salomons et Ponapé :}

Le kava a été récolté dans la province occidentale, à la frontière avec l'Irian Jaya. Cette zone de savanes arborées et de marécages est très peu propice à la culture du kava. Bien que la plante y soit connue depuis longtemps sous des noms vernaculaires locaux, elle présente tous les attributs d'une espèce introduite et cultivée dans un milieu qui lui est hostile. La plante vit rarement plus de deux ans, d'après les horticulteurs, et doit être soigneusement entretenue durant cette période. La culture fait cependant l'objet d'une commercialisation au marché du Daru. Un seul cultivar est utilisé dans cette région grande comme la Belgique. Sur la côte nord, le kava est cultivé dans la région d'Usin ainsi que sur l'île de Karkar. Le Koniak comme il est dénommé en pidgin english de cette région, fait l'objet d'une culture d'autoconsommation mais également commerciale pour approvisionner la communauté fidjienne de Lae. Les plants présentent certains caractères de dégénerescence, sont frêles et sont tous identiques (Ayou, Isa, Sipaia, Iwe). Le $P$. wichmannii pousse à l'état spontané dans cette région. Aux îles de l'Amirauté, le kava n'existe plus que sur la petite île de Baluan où il est représenté par trois cultivars dont l'un est une forme cultivée de $P$. wichmannii. Le nombre total de plants avoisine la dizaine. Il est peu probable que le $P$. methysticum existe ailleurs en Papouasie. 
Aux îles Salomon, seule Guadalcanal fut visitée, mais tous les interlocuteurs étaient unanimes pour affirmer que le $P$. methysticum $n$ 'est pas cultivé aux Iles Salomon. Par contre les formes sauvages de $P$. wichmannii sont très fréquentes en sous-bois et l'espèce est connue sous le nom vernaculaire de kwakwako.

À Ponapé, le kava est cultivé sur tout l'île et à toutes les altitudes. Il est consommé quotidiennement et lors des cérémonies coutumières. Deux cultivars sont utilisés et sont différenciés par leurs entrenœuds piquetés ou unis.

Tous les cultivars connus sous une appelation précise en langue vernaculaire de PapouasieNouvelle-Guinée, de Vanuatu, des îles Salomon, Fidji, Samoa, Tonga, Wallis, Futuna et Cook, de Tahiti et des Marquises, des îles Hawaï et de Ponapé, ont été inventoriés, récoltés et plantés en parcelles de collection. Les caractéristiques de chacun de ces cultivars ont été évaluées (tableau I). Ces plants sont décrits à l'aide de sept descripteurs morphologiques (apparence du plant : A, coloration de la tige : $\mathrm{C}$, configuration des entrenœuds : I, couleur des feuilles : L, forme du limbe : E, pubescence : $P$, profil des entrenœuds : $S$ ). Lorsque plusieurs plants ont le même codage de leur phénotype, ils se voient attribuer le même morphotype (tableau II). Du fait des échanges traditionnels de matériel végétal, il existe moins de morphotypes que d'échantillons récoltés. Les descripteurs morphologiques retenus pour identifier les morphotypes sont ceux qui permettent visuellement la discrimination la plus facile. Ils sont d'ailleurs utilisés traditionnellement par les horticulteurs. Pour cette méthode communément utilisée, seuls les caractères morphologiques permettant de différencier les cultivars.

L'absence de fertilité du kava pose la question de l'origine des très nombreux cultivars existants. Comment expliquer cette diversité si la fécondation n'existe pas? Le kava est une espèce dioïque dont le monde de reproduction est exclusivement végétatif et qui permet donc une bonne stabilité génétique des cultivars, à la mutation somatique près, mais aussi une accumulation et le maintien de l'hétérogénéité des génotypes en culture traditionnelle.

Dans l'hypothèse où des individus fertiles ont existé, le grand polymorphisme observé résulterait de fécondations croisées naturelles, donnant naissance à de multiples combinaisons des unités héréditaires. En effet, le kava étant dioïque, bien que personne n'ait jamais pu observer de pollinisation, les ovules ne peuvent être fécondés que par le pollen d'un autre plant, produisant alors de nombreux hybrides ou individus hétérozygotes résultant de parents génétiquement différents, ces derniers appartenant soit à la même espèce ( $P$. methysticum ou $P$. wichmannii) soit à des espèces proches que nous ne connaissons pas. Ces individus hétérozygotes donneraient des descendances très hétérogènes dont la variabilité s'exprime morphologiquement. Les formes observées aujourd'hui seraient issues de la conservation au cours des âges et grâce à l'homme, de la descendance de ccs fécondations croisées entre individus fertiles de ces deux binômes. Le kava serait donc un ensemble d'hybrides stériles conservés par clonage. Cependant, les observations que nous avons pu faire sur le terrain depuis 7 ans nous conduisent à considérer d'autres hypothèses.

Il ne faut pas négliger les possibilités de variation végétative pouvant être à l'origine de phénotypes très différents. En effet, chez les plupart des végétaux multipliés exclusivement par voie végétative, des variations peuvent se produire par mutation de l'ensemble, ou d'une partie, des cellules méristématiques du bourgeon et qui produisent des chimères. Messiaen (1975) a expliqué comment aux Etats-Unis un cultivar d'Ipomoea batatas appelé "Porto-Rico" avait attiré l'œil des sélectionneurs en raison de ses variations qualitatives. Rouge à chair jaune, "Porto-Rico" a donné des mutants crème à chair blanche. Messiaen explique aussi que "des mutants de rendement supérieur peuvent être obtenus par reprise permanente de la sélection clonale ". Nous avons pu vérifier nous-mêmes ces observations sur les cultivars d' $I$. batatas cultivés à la station de Tagabé (Lebot, 1986) et dont la forme du limbe pouvaient varier en l'espace de trois multiplications clonales par an. Silander (1985) décrit la formation d'une chimère produisant un individu au feuillage panaché dont les limbes sont constitués de tissus décolorés et d'autres normalement pigmentés. On ne peut s'empêcher de mentionner ici l'existence du cultivar ni kalla kiskisnian (Lebot et Cabalion, 1986), originaire de l'île de Tanna et qui correspond précisément à cette description de la chimère. L'hypothèse d'un polymorphisme d'origine asexué coïnciderait bien, nous semble-t-il avec les observations sur le terrain. Les clones peuvent selon les horticulteurs, se montrer hétérogènes après quelques "générations " (chaque bouturage) issues de ce mode de multiplication. Il ne suffit donc pas d'isoler un bon clone dans un jardin, il faut ensuite repartir, à chaque bouturage, sur des clones issus de plantes performantes et conserver en permanence les meilleures. Dans le cas du bouturage des kava, le problème posé aux horticulteurs consiste à choisir judicieusement 
Tableau I. - Zones de Prospections et de RÉCOltes

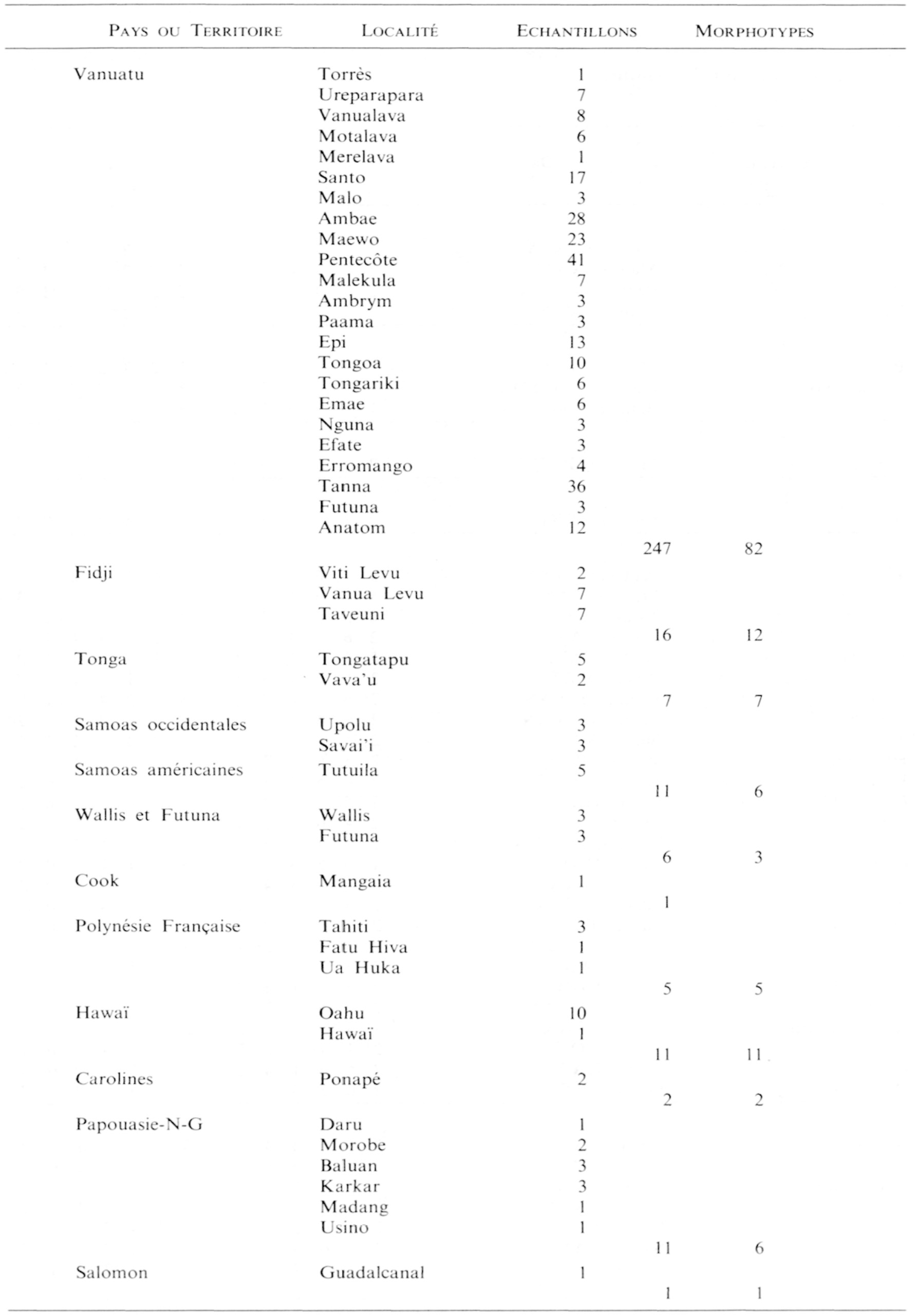


Tableau II : Regroupement des cultivars d'Océanie en fonction de leurs caractères communs, chimiques et morphologiques. 
les individus initiaux, éventuellement en éliminant les mutations désavantageuses ou encore en utilisant les mutations favorables comme point de départ de nouveaux clones.

\section{LES CONVARS SONT DES CHIMIOTYPES.}

Les données obtenues à l’aide des enquêtes ethnobotaniques nous ont indiqué l'existence de véritables "crus". L'importance des carac- téristiques chimiques des cultivars locaux est soulignée par les différents effets ressentis par les buveurs et qui chaque fois renvoient à lorigine des cultivars. Ces derniers sont, nous l'avons vu, sélectionnés d'abord pour les propriétés du breuvage quils procurent. On ne peut s'empêcher de faire la comparaison avec les travaux entrepris pour sélectionner les cépages de vigne. Comme les viticulteurs, les horticulteurs d'Océanie ont sélectionné les plants les plus vigoureux, apparemment en bonne santé 
et de productivité satisfaisante, en supprimant les moins intéressants. La sélection clonale vise à individualiser des pieds-mères, pour les multiplier ensuite séparément et former des clones qui serviront de population de base. Cette sélection a permis des progrès mais reste très empirique.

En 1966, Young et al., étudient la variabilité des six cultivars de kava à Hawaï et observent que certains d'entre eux ne diffèrent guère en ce qui concerne leur morphologie tandis que leurs teneurs et compositions présentent de grandes variations. Jossang et Molho (1970) montrent que les variations observées dans les extraits originaires de Fidji devraient être l'objet d'études plus approfondies. Enfin Duve et Prasad (1981), tentent d'évaluer la qualité d'échantillons de kava en poudre vendus sur les marchés de Fidji. Malgré un travail considérable dans ce domaine ils doivent renoncer à définir des normes pour les caractéristiques du kava. La variabilité observée par les chercheurs qui se sont intéressés au kava n'est donc pas un phénomène nouveau. Plutôt que de s'intéresser uniquement à la classique divergence morphologique, il est préférable d'étudier la divergence chimique. En effet, les teneurs et les compositions correspondent aux caractères utiles de cette culture dont le produit obtenu n'a pas de caractéristiques morphologiques mais moléculaires.

Pour cette étude de la variabilité chimique des cultivars de kava en Océanie, 322 échantillons ont été analysés et leur teneurs et compositions chimiques déterminées (Lebot et Lévesque, 1989). La composition chimique d'un échantillon est déterminée par la quantification des six kavalactones majeures qui représentent près de $96 \%$ de l'extrait concerné. La composition est exprimée à l'aide d'un code qui représente le chimiotype de l'échantillon analysé. Cette composition est codée suivant un ordre d'importance décroissante des kavalactones dans l'extrait. Par exemple, si les extraits de deux échantillons « $A$ » et « $B$ » se composent ainsi :

$\begin{array}{cc}\begin{array}{c}\mathrm{A} \\ \%\end{array} & \begin{array}{c}\text { } \mathrm{B} \text { \% } \\ \%\end{array} \\ 2 & 18 \\ 30 & 25 \\ 15 & 10 \\ 25 & 2 \\ 10 & 30 \\ 18 & 15\end{array}$

les chimiotypes codés sont :

246351521634

Chaque kavalactone est représentée par un numéro de code. Ce codage permet de se faire très rapidement une idée de la nature de la composition chimique du cultivar. Le but recherché ici est de regrouper les nombreux cultivars de kava en convars, c'est-à-dire en groupes de cultivars apparentés suivant un critère préalablement choisi. En ce qui nous concerne, il s'agit de définir des convars de chimiotypes similaires, puisque le chimiotype est le critère retenu pous cette sélection clonale.

Les données obtenues permettent de distinguer 8 grands groupes de chimiotypes en Océanie. A l'intérieur de ces grands groupes chimiques, l'utilisation des résultats obtenus lors des descriptions morphologiques, apporte des informations intéressantes. Les résultats de cette classification sont présentés dans le tableau II.

Les corrélations entre chimiotypes et morphotypes ne sont pas évidentes. Mais ce n'est pas la première fois que l'on observe " qu'il n'y a aucun parallélisme entre classification morphologique et classification chimique " (Deysson, 1967 , p. 18). Si des morphotypes identiques mais d'origines différentes peuvent présenter des chimiotypes semblables, le contraire est aussi vrai. On observe de très grandes variations de teneurs et de compositions au sein des espèces $P$. wichmannii et $P$. methysticum. Ces variations sont observées entre des formes sauvages ou cultivées, d'origines différentes et dont le polymorphisme est important, même lorsqu'elles sont plantées dans un milieu homogène. Les facteurs extrinsèques ne sont donc pas responsables de ces variations, les espèces Piper wichmannii et Piper methysticum sont composées d'individus appartenant à des chimiotypes différents et dont l'expression semble directement dépendante de facteurs intrinsèques. A ce titre, le chimiotype représente un bon indicateur de la divergence moléculaire entre les individus étudiés et un marqueur génétique intéressant pour retrouver des individus de chimiotypes apparentés situés dans des endroits différents.

Le chimiotype 521634 par exemple, n'est jamais consommé et rassemble des formes appartenant à l'espèce $P$. wichmannii. Tabal et Tangurlava ont des chimiotypes très proches de $P$. wichmannii et sont des cultivars de $P$. methysticum très peu cultivés puisque rarement consommés. Le chimiotype 256431 correspond aux cultivars dits de "deux jours". L'effet ressenti est lent mais durable. Ces cultivars sont recherchés par les buveurs soucieux d'obtenir un état d'ébrièté prolongé. Le chimiotype 265431 rassemble des cultivars à usages coutumiers et utilisés en pharmacopée traditionnelle. Les chimiotypes 246531 et 426315 rassemblent 
les cultivars utilisés quotidiennement, le dernier étant le plus apprécié. Ces cultivars ont un effet soudain, très prononcé mais éphémère. Les buveurs recherchent un fort effet physiologique qui disparaît avec le sommeil qui suit l'absorption du breuvage.

Le chimiotype 426315 correspond à un dosage naturel des kavalactones dont l'extrait aqueux présente les propriétés physiologiques les plus agréables. Cela ne surprend guère car on observe qu'un cultivar comme Kelai, qui est certainement le plus apprécié à Vanuatu, contient plus de $37 \%$ de kawaïne. Kretzschmar (1970) décrit ainsi "l'excellente activité psychopharmocaologique " de la kawaïne : "décontraction émotionnelle et musculaire, stabilisation affective et stimulation de la capacité de pensée et de la capacité d'action ". Ce genre d'explication est également valable pour des chimiotypes qui rassemblent de fortes proportions de DHM et de DHK car la plupart des auteurs s'accordent pour dire qu'il s'agit des kavalactones les plus actives. Les formes de $P$. wichmannii, Vambu, Buara, Bo et Kau en contiennent près de $75 \%$ et les cultivars de $P$. methysticum, dits de "deux jours" environ $58 \%$. Rien d'étonnant dans ces conditions, pour que les consommateurs constatent que les premiers donnent la nausée et que les derniers poursuivent leurs effets le lendemain.

Nous avons nous mêmes fait, et refait, l'expérience et constaté que ces déclarations obtenues par les enquêtes ethnobotaniques étaient parfaitement fondées. Les cultivars qui présentent des chimiotypes similaires sont très peu cultivés. Les compositions chimiques étudiées sont très nettement corrélées avec les résultats obtenus au cours des enquêtes ethnobotaniques. Elles confirment l'existence de plusieurs chimiotypes sur un même terroir et permettent de comprendre les différents effets ressentis par les buveurs. Il semble donc bien qu'il existe une adéquation entre le chimiotype et l'effet recherché par le consommateur mélanésien. Cet effet est directement corrélé la nature des kavalactones qui se trouvent en proportions majoritaires dans la racine. Cette observation n'est pas surprenante car les kavalactones majeures ont des propriétés physiologiques différentes, il est donc normal que ces compositions naturelles produisent des effets différents. Il est aussi possible d'effectuer des corrélations entre les chimiotypes et le rang des cultivars dans la classification traditionnelle. On observe que les cultivars les plus cultivés (en nombre de plants) ont toujours un chimiotype identique. Il s'agit bien sûr des cultivars consommés quotidiennement.
Les chimiotypes $\mathrm{A}, \mathrm{B}, \mathrm{C}$, et $\mathrm{D}$ sont exclusivement limités à la Mélanésie et correspondent à des formes sauvages ou cultivées de $P$. wichmannii. Le chimiotype A (521634) présente de très fortes proportions de DHM et de DHK (entre 38 et $58 \%$ ). Ces deux kavalactones représentent ensemble près de 64 à $75 \%$ du total. La proportion de $\mathrm{K}$ est très faible $(<3 \%)$. Les chimiotypes $\mathrm{B}$ et $\mathrm{D}$ ont une très forte teneur en DMY (de 25 à $35 \%$ ) et une très faible proportion de $K$. Le chimiotype $C$ présente des proportions équivalentes de DHK, DHM et de $\mathrm{M}(31 / 30 / 27)$ et presque pas de $\mathrm{K}$ $(0,99)$. Tous sont des variants de $P$. wichmannii et sont caractérisés par une très faible teneur en $\mathrm{K}$, la kavalactone la plus intéressante pour le buveur. Les morphotypes de ces individus sont différents mais tous ont un port érigé et produisent peu de tiges.

Les chimiotypes E, F, G, H et I rassemblent des cultivars de $P$. methysticum. Le chimiotype $E$ produit un breuvage dont l'effet physiologique, très fortement ressenti, résulte des fortes proportions de DHM et DHK. Ce chimiotype est présent à Vanuatu, Tonga, Wallis, Fatu hiva, Oahu et Ponapé.

Le chimiotype $F$ est proche du précédent mais présente de plus fortes proportions de DHK et de DHM est une très faible teneur en Y. Ce chimiotype n'existe qu'à Vanuatu et en Papouasie-Nouvelle-Guinée.

Le chimiotype $G$ rassemble tous les cultivars utilisés pour la consommation quotidienne, plus particulièrement dans les îles où les racine est préparée fraîche.

Le chimiotype $\mathrm{H}$ est très certainement celui qui procure le breuvage le plus apprécié en raison de sa forte teneur en $\mathrm{K}$, plus de $37 \%$ dans le cas de kelaï. Il n'existe qu'à Vanuatu et à Samoa.

Le chimiotype I n'est présent que dans les îles où le kava est préparé à partir de la racine sèche. Il présente une très forte proportion de M (méthysticine), la moins soluble des kavalactones, et qui lors de la préparation du breuvage est probablement extraite dans de moindres proportions.

Nous avons représenté (figure 1) la généalogie des chimiotypes qui conduit des formes sauvages de $P$. wichmannii aux formes cultivées de $P$. methysticum, en tenant compte des corrélations entre les différentes kavalactones. On observera l'amélioration progressive du caractère "forte teneur en kawaïne ${ }^{4}$ et faible teneur en dihydrométhysticine ${ }^{5} "$. Cette sélection généalogique et clonale sur des plants individuels est faite empiriquement par les horticulteurs à chaque récolte de plant et après 
avoir consommé le breuvage. Le processus de domestication vise donc à sélectionner au sein du clone les replicants du chimiotype, s'il y a lieu de le conserver, ou de sélectionner des variants du chimiotype, si leurs caractéristiques s'avèrent plus intéressantes. Il existe un bon nombre d'arguments qui permettent d'affirmer que Vanuatu, centre de diversité incontestable, est aussi le centre d'origine du $P$. methysticum. Cette hypothèse de filiation entre une forme sauvage et les cultivars d'aujourd'hui n'est valable que pour le cas de Vanuatu, elle serait différente à partir d'une forme sauvage de Papouasie ou des Iles Salomon. On observe en effet une très grande variabilité chimiotypique chez $P$. wichmannii. Ce qui nous semble normal pour une espèce dioïque fertile.

\section{Conclusions.}

Au terme de cette étude, nous constatons qu'aucune des informations recueillies à l'aide de l'une des approches utilisées ne contredit celles obtenues par une autre. Les hypothèses formulées en ethnobotanique restent acceptables et tangibles en botanique et en chimie, et vice versa. Les conclusions dont nous fait bénéficier une discipline contribuent grandement à expliquer les résultats obtenus par ailleurs. Les résultats obtenus au cours de ces travaux, corrélés aux données obtenues par les Herbiers concernés, par la littérature et par les enquêtes etnobotaniques, nous permettent de tirer les conclusions suivantes :

Les kavas en Océanie correspondent à deux binômes : $P$. wichmannii, qui rassemble les formes sauvages séminifères et $P$. methysticum, qui rassemble des cultivars aspermes. La présence du $P$. methysticum en un endroit résulte de l'intervention de l'homme qui le multiplie exclusivement par voie asexuée. Cette plante disparaît dès qu'elle n'est plus cultivée sous l'effet conjugué des prédateurs, des pilleurs et de la sénescence.

Pour une espèce à multiplication végétative exclusive, le polymorphisme observé est impressionnant mais n'est pas surprenant lorsque l'on compare le kava aux autres cultures. Les observations réalisées sur le terrain incitent à penser que les nombreux morphotypes de kava ont deux origines possibles. La variabilité décrite aujourd'hui résulte de fécondations croisées intervenues dans le passé et conservées grâce à l'homme. Mais cette variabilité n'est pas définitive car elle résulte aussi probablement de mutations somatiques qui attirent l'œil des horticulteurs-sélectionneurs. Ces hypothè- ses ne sont pas contredites par l'ethnobotanique et suggèrent que les ressources génétiques du kava puissent êtres conservés in situ.

Pour cette étude du kava sur la totalité de son aire de distribution, plus de 118 cultivars, correspondants à autant de morphotypes, ont été récoltés dans 42 îles du Pacifique et plantés en collections vivantes. Les descriptions morphologiques ne sont pas suffisamment discriminantes pour regrouper les cultivars mais les informations recueillies permettent de compléter celles obtenues par les analyses chimiques et d'établir ainsi un code d'identité permettant de reconnaître deux cultivars d'appellations et d'origines différentes. Les analyses chimiques et les essais que nous avons réalisées montrent que le kava rassemble plusieurs chimiotypes et que le chimiotype peut être considéré comme étant l'expression chimique du génotype. Les cultivars et les formes sauvages d'Océanie se regroupent en 8 grands chimiotypes qui présentent chacun des caractéristiques chimiques distinctes et qui sont responsables d'effets physiologiques différents. La variabilité du caractère utile du kava, le chimiotype, est traditionnellement utilisee pour satisfaire différents usages.

Ces résultats ont également démontré l'efficacité de la chimiotaxonomie. Deysson (1967) a observé que « les caractères chimiques devraient être, en principe, très utiles pour la définition des espèces dans la mesure où ils permettent de dégager les caractéristiques fondamentales d'un métaboíisnie $\%$. Les chimiotypes sont comme nous l'avons vu, très utiles pour différencier les formes de $P$. wichmannii et de $P$. methysticum.

Lorsque deux appellations différentes, récoltées en deux endroits différents, présentent les mêmes chimiotypes et les mêmes morphotypes, il y a lieu de penser qu'il s'agit d'un seul et même cultivar. Piper methysticum n'est représenté que par un seul cultivar sur la côte nord de la Papouasie-Nouvelle-Guinée (Usino, Morobe, Madang et Karkar). Ce cultivar ne semble pas exister ailleurs. Le seul cultivar existant dans la vaste province occidentale de la Papouasie est très proche du cultivar nommé Malmalbo, originaire de l'île de Pentecôte à Vanuatu.

Bien que de très grandes distances géographiques séparent les cultivars polynésiens, les distances phénotypiques et chimiotypiques sont très courtes et permettent de rapprocher les cultivars de cette zone. Ainsi, le cultivar Omoa, originaire des îles Marquises semble très proche de cultivar Oahu 24I, originaire des îles Hawaï. Des observations similaires peuvent être faites pour d'autres cultivars : par exemple, le culti- 
var Aigen, originaire de l'île de Tanna au sud de Vanuatu semble identique au cultivar $A v a$ $u l u$, originaire de l'île de Tutuila aux Samoas américaines. Bien qu’à Tanna, la tradition orale rapporte que le kava fut introduit depuis Tonga, les vecteurs de cette introduction sont probablement les polynésiens des petites îles d'Aniwa et de Futuna, qui d'après certains linguistes parlent une langue très proche du Samoan (Clark, 1979). En Polynésie centrale, il apparaît très nettement qu'un échange intensif de matériel végétal a eu lieu entre les îles Fidji, Tonga, Wallis et Samoas.

En Papouasie-Nouvelle-Guinée, tous les cultivars de $P$. methysticum se regroupent en un seul convar, quelles que soient leurs origines géographiques (région de la Fly, côte nord, île de Karkar et île de Baluan), alors que ces régions sont situées à plusieurs centaines de $\mathrm{km}$ de distance. Ces résultats montrent que les chimiotypes de ces cultivars sont très proches et leur variabilité quasi nulle, ce qui semble indiquer une introduction récente de matériel végétal peu diversifié. Il en va de même pour les formes sauvages ou cultivées de $P$. wichmannii qui présentent une nette homogénéité chimiotypique. La situation est fort simple aux îles Salomon avec deux formes sauvages de $P$. methysticum de Ponapé constituent un groupe à part qui s'associe à une forme sauvage de $P$. wichmannii de l'île de Karkar en PapouasieNouvelle-Guinée et à un "kava sauvage", originaire de la région de Lae. que je n'ai pu récolter mais que m'a expédié le Dr. Thredgold de l'Université de Lae.

Une espèce cultivée provient généralement de la domestication d'une espèce sauvage. Son aspect est généralement très différent puisque l'objectif de la domestication est de modifier les caractères sauvages pour les adapter aux besoins de l'homme. Chez le kava, les caractères intéressants ne sont pas visibles parce qu'il s'agit des propriétés chimiques. Il n'y a donc aucune raison pour que l'espèce sauvage soit vraiment différente morphologiquement de l'espèce cultivée. L'évolution des caractères se fait sous l'effet des facteurs du milieu et sous la pression de la sélection exercée par l'homme. Comment expliquer, si ce n'est par le processus de la sélection, que le kava soit l'une des plantes médicinales dont le rendement en matière active soit le plus élevé. Les kavalactones n'ont pas de fonction apparente dans le métabolisme du kava, leur rôle physiologique demeure énigmatique. Il est difficile de dire quel est leur rôle écologique, par exemple dans les mécanismes de défense contre les prédateurs. Ces derniers ont été introduits en Océanie par l'homme, bien après les premières utilisations du kava. Bon nombre d'insectes attaquent le kava sans être affectés par la présence des lectones. La partie souterraine de la plante joue ici un rôle de stockage de ces métabolites, probablement synthétisées directement dans les racines comme chez beaucoup de plantes médicinales. Jossang et Molho (1970) ont d'ailleurs montré que les feuilles ne contenaient que deux kavalactones (DHK et DHM) et Smith (1979) a identifié un alcaloïde présent dans les feuilles mais absent dans les racines.

Peu de plantes connaissent une aussi forte pression de la sélection humaine qui s'exerce à chaque récolte individuelle de plant et qui vise l'amélioration constante des caractères utiles, c'est-à-dire des teneurs et compositions adéquates. Le caractère utile du kava est la présence des kavalactones. Selon Sengupta et Ray (1987) qui ont effectué un inventaire exhaustif des composés isolés de toutes les espèces du genre Piper, les kavalactones n'existent que chez $P$. methysticum. L'ancêtre du kava est donc bien le kava des ancêtres, le $P$. wichmannii et son aire d'origine se situe très certainement sur l'aire de distribution de cette dernière espèce. Or le $P$. wichmannii n'existe nulle part ailleurs qu'en Mélanésie. Reste donc à préciser où en Mélanésie a eu lieu la domestication.

Le $P$. methysticum existe en Papouasie-Nouvelle-Guinée mais en des endroits très localisés et sa variabilité est quasi nulle ce qui semble indiquer une base génétique originelle très limitée et une culture récente à l'échelle humaine. Comme le $P$. methysticum n'existe pas aux lles Salomon, on peut à la limite supposer qu'il y a été cultivé un jour, bien que rien dans la littérature ne l'indique, ou qu'il a disparu, ou encore qu'il n'a jamais existé dans ces îles, ce qui nous paraît plus probable. La présence du kava en Papouasie-Nouvelle-Guinée et aux Iles Salomon résulterait dans ce cas d’un phénomène de retour opéré par les Polynésiens, ce qui expliquerait qu'il soit très localisé et exclusivement limité aux côtes dans le premier cas et aux colonies polynésiennes (Vanikoro, Tikopia) dans le second.

Le kava est dans ce cas originaire du nord de l'archipel de Vanuatu où les horticulteurs entretiennent encore tous les individus qui résultent du processus de domestication. Plusieurs arguments sont en faveur d'une origine unique du kava plutôt que des découvertes simultanées ou éparses géographiquement. Les rites et usages, les mythes et légendes, les noms vernaculaires enfin, confirment que les boutures ont voyagé de main à main comme l'on 
donne un bâton de relais mais avec leur nom et leur mode d'emploi. La plante a très certainement été introduite partout où l'homme souhaitait s'installer. Il n'existe pas d'autres espèces en Océanie qui permettent à l'homme cet accès convivial aux paradis artificiels, mieux vaut donc ne pas s'en démunir. Comme le kava ne peut être dispersé par ses fruits, l'homme en est le vecteur.

Le kava est une espèce dont l'aire de distribution est exclusivement limitée à l'Océanie. C'est la seule espèce cultivée de cette importance économique dans ce cas. Cette situation résulte du fait que l'aire d'origine de l'espèce sauvage est située en Papouasie alors que la dispersion géographique des autres espèces cultivées est beaucoup plus vaste. Cette observation d'ordre biogéographique est reprise à bon compte par les décideurs qui voient là une occasion unique de contrôler les cours diune denrée dont ils ont l'exclusivité par opposition aux autres cultures d'exportation. Sans porter de jugement dappréciation sur cette idée, il convenait tout de même d'entraver l'érosion génétique de cette espèce, initiée à l'ère européenne. Nous l'avons vu, 21 cultivars existaient aux Marquises, 14 à Tahiti et au moins autant à Hawaï. Ils ont disparu à jamais et avec eux des compositions chimiques certainement très intéressantes. Si nous n'avons pu les ressusciter, au moins avons nous pu préserver la variabilité existante.

Il est probable que le plus ancien foyer de l'agriculture se situe en Papouasie-NouvelleGuinée et non pas en Mésopotamie, si l'on accepte que l'horticulture est une forme d'agriculture (Bellwood, 1981, Yen, 1988). L’étude de la domestication des espèces cultivées originaires du foyer horticole indo-océanien, ainsi que de leur distribution dans le temps et l'espace, est une approche intéressante pour préciser, à l'aide de méthodes diverses mais complémentaires, les processus de domestication. Les îles océaniques du Pacifique sont des laboratoires particulièrement bien adaptés à ce genre d'exercice puisqu'elles constituent une zone géographique où toutes les cultures ont été importées.

\section{BIBLIOGRAPHIE}

A.C.C.T. - 1977. Dictionnaire d Agriculture. La Maison Rustique. 220 P.. Paris

Ashry G. - 1987. A guide to Pohnpei. An island Argosy. Rainy Day Press. Oregon. 314 p.

BARRAL J. - 1956. Lagriculture vivrière indigène aux Nouvelles-Hébrides. J. S. Oc.. N" spécial. XII : (204).
BARral J. 1957. À propos du Piper methisticum. JATBA. IV. 5-6: 270-273.

Bakral J. -- 1957. Laurbre à pain en Océanie. J. Soc. Oc. 4 : 117-123.

Barral J. - 1957. Lënigme de la patate douce en Océanic. Etudes d'Outre-Mer, 40. (4).

Barral J. - 1962. Les plantes alimentaires de locéanic. origines. distribution. et usages. Thèse de Doctorat. Faculte des Sciences. Marseille.

Brlewood P. -- 1981. Le peuplement du Pacifique. Pour la Science. N" 39. janv.

Boninemaison J. - 1985. Les fondements d'une identité. Territoire. histoire et société dans larchipel de Vanuatu (Mèlanésie). Thèse Doctorat. Univ. Paris IV., ORSTOM.

Bonifmaison J. - 1986. La dernière ile. Éd. Altea-ORSTOM. Paris.

Bolrgaril A. - 1865. Des races de l'Océanie française. de celles de la Nouvelle-Calédonie. Mém. Soc. Anthrop. Paris. 2“ part. : 403.

BraldeNblikg W.A. - 1985. Classification of cultivated plants. Acta Horticulturae. First Intern. Symp. on Taxonomy of cultivated plants. Van der Maesen ed.. 109-115.

Brossis C. - 1756. Histoire des navigations aux mers australes. Durant ed. 3 vol.. 370-389. Paris.

BROW: (F.B.H.) - 1935. FLORA OF SOLTH EASTERN POLYNesia. III. Dicotyledons. B. P. Bishop MLS. BLll., 130 : PIPr.k, 17-20.

ChI:W Wfe-LeK - 1972. The genus Piper (Piperacese') in New Guinea. Solomon Islands and Australia. J. Arnold Arb., $53: 1-25$.

ClakK R. - 1979. Languages. In Jennings J. D. ed. The Prehistory of Polynesia. Cambridge. Massasuchetts. Harvard University Press.

Codrington R. H. - 1981. The Melanesians. Oxford. Clarendon Press.

COOK R. E. -- 1985. Growth and Development in Clonal Plant Populations. In Population Biology and Evolution of Clonal Organisms. PP. 259-296. Yale University Press. New Haven and London.

ClzhNT G. - 1856. Du Kawa de Noukouhiva. lles Marquises. Rev. Col.. Paris. 2“ séric. 15 : 582-583.

Clzant G. - 1857. Du kawa ou ava de Tahiti (Piper methisticum). Le messager de Tahiti. Papeete. 10 mai et 29 novembre.

Deginfr O. - 1940. Flora Hawaïensis. Vol. 4. Honolulu.

DE LESSERT B. - 1837. Icones Selectae Plantarum. Vol. III. Tab. 89.

DEysSON G. -- 1967. Organisation et classification des plantes vasculaires. SEDES, Paris.

Dive R. N. et PrasAd J. - 1981. Quality evaluation of Yagona (Piper methysticum). Fidji Agric. J. 46. 1 : 5-9.

FIRTH R. - 1954. Anuta and Tikopia : symbiotic elements in social organisation. J. Pol. Soc.. 63:87-131 (p. 102).

ForNANder A. - 1919. Hawaïan antiquities and folklore. Memoirs. B. P. Bishop Mus.. VI. Honolulu.

Forster J. G. A. -- 1786. De Plantis Esculentis Insularum Oceani Australis. 80 p.. Berlin.

Fox C.F. - 1924. The threshold of the Pacific. Londres.

Garangi: J. - 1972. Petite histoire dune préhistoire celle des Polynésiens. J. Soc. Oc. 74-75.

Gatty R. - 1956. Kava-Polynesian beverage shrub. Econ. Bot.. X : 241-249. 
Hansel R. - 1968. Characterization and physiological activity of some kava constituents. Pacific Science, 22, 3 : 293-313.

Harlan J. R. - 1970. The evolution of cultivated plants. In Frankel and Bennett Ed. "Genetic Resources in Plants, their exploration and conservation ". Blackwell Scientific Publ.. Oxford.

Hacdricolirt A. G. et Hedin L. - 1943. L’homme et les plantes cultivées. Paris.

Heinlein M. el al. - 1984. Wilt and stem rot control in Yagona. Dept. Agric., Fiji, Annual Report, (51-52).

Hocart A.M. - 1952. The northern States of Fiji. Londres.

HoRnell J. - 1946. How did the sweet potato reach Oceania. Journal of the Linnean Soc. of London. 53, 348.

Hotgh G. - 1905. Kava drinking as practised by the Papuans and Polynesians. Smithsonian miscellanous collections 47 : 85-92.

Jossang P. et Molmo D. - 1970. Études des constituants des feuilles de Piper methysticum Forst. Bull. Mus. Hist. Nat., sér. 3, 42, 2 : 440-447.

Koenig et al. - 1986. Préface, in Plantes utiles de Polynésie Française, Rau Tahiti. P. Pétard. Edition revue et illustrée. Haere Po No Tahiti. Papeete.

LARSEN A. - 1985. Notes on root crops in Vanuatu. Fao, Suva, Fiji.

Lebot V., Cavalion P. et Levesque J. - 1986. Le kava des ancêtres ( $P$. wichmannii $C$. DC.) est-il l'ancêtre du kava ( $P$. methysticum Forst.)? Naika (VNSS), $23: 1-10$.

Lebot V. et Cabalion P. - 1986. Les Kavas de Vanuatu (cultivars de Piper methysticum Forst.). Coll. Trav. Doc. ORSTOM n" 205, Paris.

Leвот V. - 1986. Evaluation of local and introduced cultivars of Sweet Potato (Ipomoea hatatas (L.) Lam.) in Vanuatu. Alafua Agric. Bull. 11 (3) : 25-31.

Lebot V. - 1986. Les kavas en Océanie (Piper methysticum Forst. f, et Piper wichmannii C. DC.), étude pluridisciplinaire d'une culture traditionnelle. Thèse de Doctorat, Faculté des Sciences, Montpellier.

Lebot V. et LeVesqle J. - 1989. The origin and distribution of kava (Piper methysticum) : a phytochemical approach. Allertonia. vol. 5, n" I, Pacific tropical batanical garden, Hawaï.

Lester R. H. - 1941. Kava drinking in Viti Levu, Fiji. Oceania, $12,3: 226-254$.

Liwin L. - 1927. Phantastica. Petite bibliothèque Payot, Paris, réed. 1970.

Mac'Daniels L. H. - 1971. A study of the Fe'i banana and its distribution with reference to Polynesian migrations. B.P. Bishop Mus. Bull. p. 190.

Marshall W. - 1976. A review and appraisal of alcohol and kava studies in Iceania. In Cross-cultural approaches to the study of alcohol : an interdisciplinary perspective. World Anthropology series, La haye, p. 103-118.

Messiaen C. M. - 1975 Le Potager tropical. Tome 3. Cultures spéciales. Presses Universitaires de France et A.C.C.T. Paris.

MicklolCho-MaClay N. VON - 1886. List of plants in use by the natives of New Guinea. Proc. Linn. Soc. N.S.W. for 1885. 10 : p. 346-358.

Nevermani H. - 1938. Kawa auf Neu Guinea. Ethnos, 3 : p. 179-192.

Parham J. W. - 1964. Plants of the Fiji Islands. 221, Fig. 78.
Parkinson S. - 1773. A journal of a voyage to the south seas in his Majesty's ship the Endeavour (1768). Londres p. 37.

Riesenberg S. H. - 1968. The native policy of Ponape. Smithsonian Institute Press. Washington.

Rivers W. H. R. - 1914. The history of Melanesian Society. 2 vol., Cambridge.

Samcel R. - 1986. Chromosome numbers in Piper. Kew Bull. 42 (2) : p. 465-470.

SAÜER H. et HäNSEL R. - 1967. Kawalactone und flavonoide aus einer endemischen Piper-Art Neu Guineas. Planta Medica, 15, 4 : p. 443-458.

Seemann B. - 1865. Piperaceae in Flora Vitiensis Nova. Reeves, Londres : p. 259-262.

Sengupta S. et Ray A. B. - 1987. The chemistry of Piper species : a review. Fitoterapia, vol. LVIII, n" 3 : p. 147. 166.

Silanider J.A. - 1985. Microevolution in Clonal Plants. In Population Biology and Evolution of Clonal Organisms. Yale University Press. New Haven and London : p. 107-152.

Smith A. C. - 1981. Flora Vitiensis Nova. Vol. 2, 810 p., Hawaï, p. 58-59.

Sprigis M. - Early coconut remains from Aneityum Islmand, southern Vanuatu, S. W. Pacific. Bulletin of Indo-Pacific Association. A.N.U. Canberra. 1982

Steinmetz E. F. - 1960. Piper melhysicicum (kava) Famous drug plant of the South Sea Islands. Amsterdam, p. 46.

OSTERLY J. - 1967. Kawa in Melanesia. Ethnos, 32 : p. $97-$ 121.

Sterly J. - 1970. Heilpflanzen der Einwohner Melanesiens. Beiträge zur Ethnobotanik des Sudwestlichen Pazifik. Hamburger Reihe zur Kultur - und Sprachwissenschaft. Hamburg.

Thompson B. - 1908. The Fijiamns : a study of the decay of custom. Londres : p. 213, 341-351.

Тıтсомв M. - 1948. Kava in Hawaï. J. Pol. Soc., 5 : p. 105-201.

VAVILOV N. I. - 195I. The origin, variation, immunity and breeding of cultivated plants. in Selected writing of N. 1 . Vavilov. Traduit par K. Starr Chester, Chronica bot. 13. p. 1-364.

WII.JFR G. P. - 1928. The Bread - Fruit in Tahiti. Bernice P. Bishop Museum Bulletin 50, Honolulu.

Williams T. et Calvert J. - 1859. Fiji and the Fijians. D. Appleton and Compagny. New York.

Williams F. E. - 1940. Drama of Orokolo: the social and ceremonial life of the Elema. The Clarendon Press. Oxford.

WoDzıckı K. - 1979. - Relationships between rats and man in the central P«acific. Ethnomed. V, 3/4: p. 433446.

YEN D. E. - 1974. The sweet potato and Oceania. Bernice P. Bishop Museum Bulletin $n^{\circ}$ 236, Honolulu.

YEN D. E. - 1988. Polynesian cultigens and cultivars : the questions of origin. Communication présentée à Laie. Oaly le 2 déc., Workshop on Ethnobotany, Brigham Young University.

Young R. L. et al. - 1966. Analysis for kawa pyrones in extracts of Piper methysticum. Phytochem., 5. 4 : p. 795798.

Yuncker T. G. - 1959. Piperaceue of Micronesia. Occas. Papers B. P. Bishop Mus., XXII, 8 : p. 88-89. 